\title{
Article
}

Mycosphere

\section{Extensions of known geographic distribution of aphyllophoroid fungi (Basidiomycota) in Finland}

\author{
Kunttu $P^{1}$, Kulju $M^{2}$, Kekki $T^{3}$, Pennanen $\mathbf{J}^{4}$, Savola $\mathrm{K}^{5}$, Helo $\mathrm{T}^{6}$ and Kotiranta \\ $\mathbf{H}^{7}$
}

${ }^{1}$ University of Eastern Finland, School of Forest Sciences, P.O. Box 111, FI-80101 Joensuu, Finland

${ }^{2}$ Biodiversity Unit P.O. Box 3000, FI-90014 University of Oulu, Finland

${ }^{3}$ Jyväskylä University Museum, Natural History Section, P.O. BOX 35, FI-40014 University of Jyväskylä, Finland

${ }^{4}$ Pentbyntie 1 A 2, FI-10300 Karjaa, Finland

${ }^{5}$ The Finnish Association for Nature Conservation, Itälahdenkatu 22 b A, FI-00210 Helsinki, Finland

${ }^{6}$ Erätie 13 C 19, FI-87200 Kajaani, Finland

${ }^{7}$ Finnish Environment Institute, P.O. Box 140, FI-00251 Helsinki, Finland

Kunttu P, Kulju M, Kekki T, Pennanen J, Savola K, Helo T, Kotiranta H 2016 - Extensions of known geographic distribution of aphyllophoroid fungi (Basidiomycota) in Finland. Mycosphere 7(3), 333-357, Doi 10.5943/mycosphere/7/3/7

\begin{abstract}
This article contributes the knowledge of Finnish aphyllophoroid funga with nationally or regionally new species, and records of rare species. Ceriporia bresadolae, Clavaria tenuipes and Renatobasidium notabile are presented as new aphyllophoroid species to Finland. Ceriporia bresadolae and $R$. notabile are globally rare species. The records of Ceriporia aurantiocarnescens, Crustomyces subabruptus, Sistotrema autumnale, Trechispora elongata, and Trechispora silvaeryae are the second in Finland. New records (or localities) are provided for 33 species with no more than 10 records in Finland. In addition, 76 records of aphyllophoroid species are reported as new to some subzones of the boreal vegetation zone in Finland. Notes on substrata and habitats of each record are given, and the ecology and distribution of some species are discussed.
\end{abstract}

Key words - aphyllophorales - biogeography - boreal forest - clavarioids - corticioids polypores - wood-inhabiting fungi

\section{Introduction}

Aphyllophoroid fungi are non-gilled, ecological form group of the Basidiomycota. They comprise various kinds of fungi, such as corticioids, polypores, clavarioids and tremelloids. These groups are highly diverse and polyphyletic (Hibbett et al. 2014). In Finland, 86.5\% of aphyllophoroid fungi grow in various kinds of forests and $75.1 \%$ are decayers (Kotiranta et al. 2009). In addition to wood decayers, aphyllophoroid fungi also include litter decayers, ectomycorrhizal species, parasitic fungi and mycoparasites (Kotiranta et al. 2009). Aphyllophoroid fungi, as wood-decaying fungi in general, play ecologically the key role in forest ecosystems by decomposition of dead wood and by this way they take care of the carbon and nutrient cycles of the forests (Boddy et al. 2008). Fungi also provide microhabitats and nutrition for wide range of invertebrates, slime moulds and parasitic fungi (Jonsell et al. 2001, Dudka \& Romanenko 2006, Boddy et al. 2008). In this paper we consider mainly corticioids, polypores and clavarioids. 
The first Finnish checklist of aphyllophoroid fungi was published in 2009, containing 980 species of which 756 corticioid and polypore species (Kotiranta et al. 2009). After this, 32 species new to Finland have been found, and plenty of biogeographical knowledge has accumulated especially on polypores and corticioids, but little also on clavarioids (e.g. Kunttu et al. 2010, 2011, 2012, Miettinen et al. 2012, Niemelä 2012, Kotiranta \& Larsson 2013, Kotiranta \& Shiryaev 2013, Spirin et al. 2013, Juutilainen 2016). The occurrence and distribution of many aphyllophoroid fungal species are still inadequately known in Finland. Thus there are still large gaps in knowledge of their ecology, biogeography, substrata and habitat requirements. The reasons behind this are time-consuming sampling, small basidiomata which are tricky to find, narrow ecological niches (substratum), unstable taxonomy and challenges in species identification.

In this article we present new records of aphyllophoroid fungi in Finland. These records derive from the field studies conducted in different parts of Finland, but also sporadic records were included. We consider three kinds of records: species new to Finland, rare or seldom collected species with maximum ten previous records in Finland, and species which are new to some subzone (section) of the boreal vegetation zone in Finland.

\section{Materials \& Methods}

The records in the current article were made mainly during the years 2008 to 2015, but some older records are also included. The field work methods of inventories varied to some extent. Many records were made in nature conservation areas as a result of species diversity inventory. Some records were made at the same time with other outdoor activities or nature inventories as a result of random sampling of species. Also surveys of species assemblages or biogeographical research were carried out in certain areas. Often used inventory method was opportunistic sampling of species (Stokland \& Sippola 2004), but for example on the island of Örö in the Baltic Sea, the inventory method was a systematic survey of certain number of drift wood logs.

In presented records, we use three levels for the names of sites: municipality, village / large nature conservation area and topographical name. For coordinates, we use the Finnish National Uniform Coordinate System (UCS, $27^{\circ}$ E) according to Heikinheimo \& Raatikainen (1981). Biogeographical provinces (at the beginning of each record) are according to Knudsen \& Vesterholt (2008), the Latin province names are presented on the website of the Finnish Museum of Natural History (Anonymous 2014). The map showing the forest vegetation zones and their subzones (Fig. 1) was modified from Rassi et al. (2010). In accordance with the above-mentioned sources, names of the subzones are presented in English (e.g. Ostrobothnia), contrary to the Latin names of the biogeographical provinces (e.g. Ostrobottnia).

Followed taxonomy and nomenclature are mainly according to Kotiranta et al. (2009), but in case of some species Miettinen \& Larsson (2011), Bernicchia \& Gorjón (2010), and Niemelä (2012) were used. The nomenclature of the genus Hyphodontia sensu lato follows Hjortstam \& Ryvarden (2009). The Finnish national red-list evaluation of the IUCN standard is according to Kotiranta et al. (2010). The decay stage classification (1-5) of dead wood is according to Renvall (1995), where stage 1 means hard, hard dead wood and 5 means completely decayed wood. Diameter of dead wood was measured at breast height if the trunk was complete, and in the middle if the trunk was broken. We use the term "kelo" referring to dead and old-growth trunks of Scots pine with a grey and decorticated surface. Scots pine can become kelo trees mainly in dry and barren forest habitats (Leikola 1969, Niemelä et al. 2002).

The material was collected, identified and confirmed by several researchers mentioned in the record information. The most often mentioned researchers are referred to the following abbreviations: HK = Heikki Kotiranta, JP = Jorma Pennanen, KS = Keijo Savola, MK = Matti Kulju, PH = Pekka Helo, PK = Panu Kunttu, TH = Teppo Helo, and TK = Tapio Kekki. The collector is also the identifier unless otherwise stated. The code after the collector's name or abbreviation is a personal sampling number of the specimen. Voucher specimens are deposited in the herbaria of the universities of Helsinki (H), Oulu (OULU), Turku (TUR), Bratislava (BRA), and/or in the private reference herbarium of Jorma Pennanen (J.P.), Keijo Savola (K.S.), and Heikki Kotiranta (H.K.). 


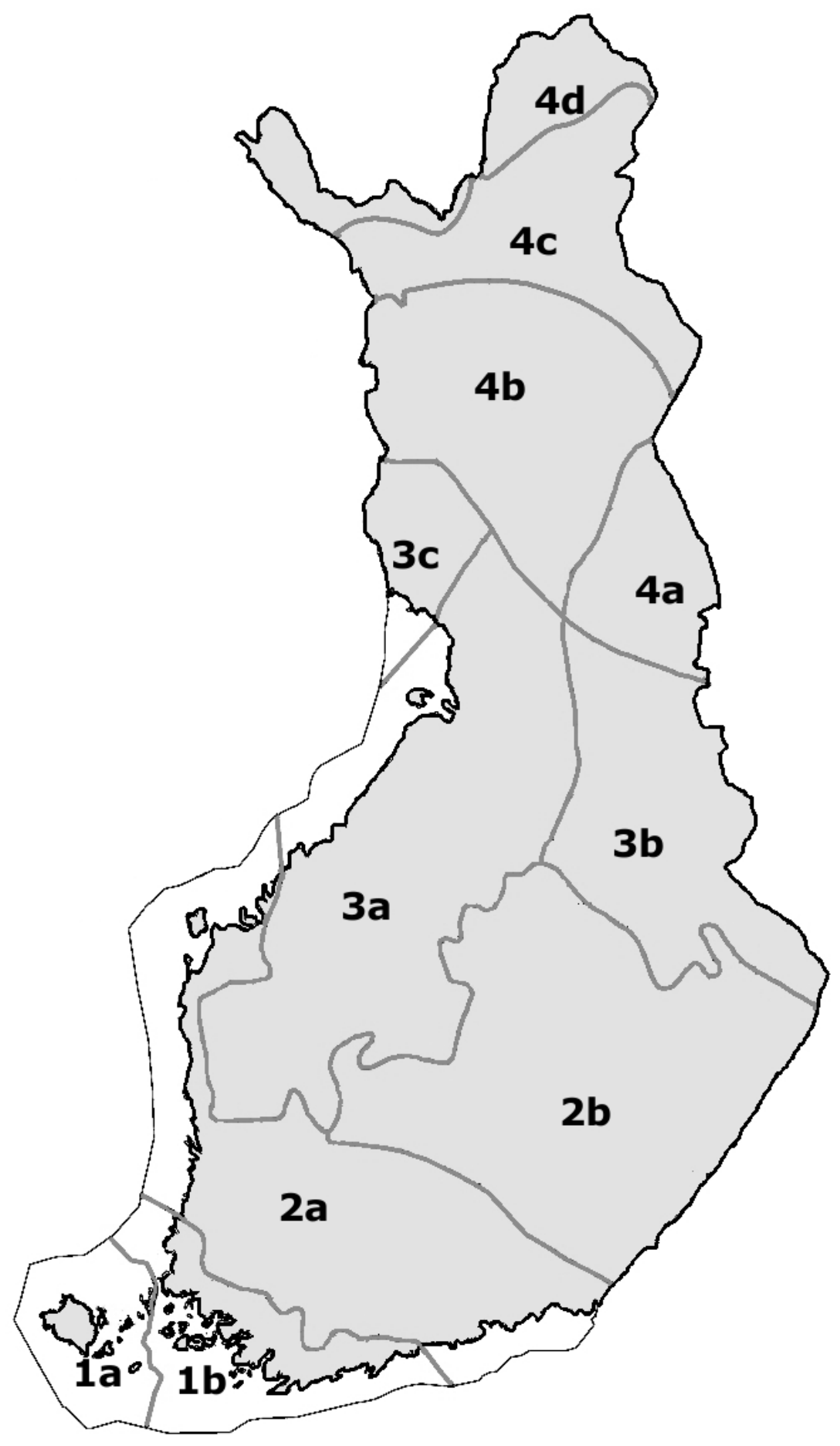

Fig. 1 - Boreal forest vegetation zones (1-4) and their subzones in Finland. 1a - Hemiboreal, Åland; $1 \mathrm{~b}$ - Hemiboreal, Oak Zone; $2 \mathrm{a}$ - Southern Boreal, Southwestern Finland and Southern Ostrobothnia; $2 b$ - Southern Boreal, Lake District; 3a - Middle Boreal, Ostrobothnia; $3 b$ - Middle Boreal, Northern Carelia - Kainuu; 3c - Middle Boreal, Southwestern Lapland; 4a - Northern Boreal, Kuusamo District; 4b - Northern Boreal, North Ostrobothnia; 4c - Northern Boreal, Forest Lapland; 4d - Northern Boreal, Fjeld Lapland.

\section{Results}

Ceriporia bresadolae, Clavaria tenuipes and Renatobasidium notabile are presented from Finland for the first time. Ceriporia bresadolae and $R$. notabile are globally very rare species. New records (localities) of the following 33 rare or infrequently collected species (in parentheses the 
ordinal number of these records in Finland): Albatrellus citrinus $\left(4^{\text {th }}-5^{\text {th }}\right)$, Antrodia cretacea $\left(3^{\text {rd }}\right)$, Antrodia leucaena $\left(3^{\text {rd }}-4^{\text {th }}\right)$, Antrodia mappa $\left(5^{\text {th }}\right)$, Antrodia piceata $\left(5^{\text {th }}-8^{\text {th }}\right)$, Ceriporia aurantiocarnescens $\left(2^{\text {nd }}-3^{\text {rd }}\right)$, Chaetoporellus curvisporus $\left(7^{\text {th }}\right)$, Clavaria flavipes $\left(3^{\text {rd }}-10^{\text {th }}\right)$, Clavaria greletii $\left(6^{\text {th- }} 8^{\text {th }}\right)$, Colacogloea peniophorae $\left(10^{\text {th }}\right)$, Crustomyces subabruptus $\left(2^{\text {nd }}\right)$, Gloeophyllum carbonarium $\left(9^{\text {th }}-10^{\text {th }}\right)$, Luellia recondita $\left(6^{\text {th }}\right)$, Paullicorticium pearsonii $\left(5^{\text {th }}\right)$, Phanerochaete livescens $\left(3^{\mathrm{rd}}\right)$, Phlebia subcretacea $\left(9^{\text {th }}\right)$, Pycnoporellus alboluteus $\left(8^{\text {th }}\right)$, Resinicium pinicola $\left(4^{\text {th }}\right)$, Scytinostromella nannfeldtii $\left(4^{\text {th }}\right)$, Sidera vulgaris $\left(6^{\text {th }}-8^{\text {th }}\right)$, Sistotrema autumnale $\left(2^{\text {nd }}\right)$, Sistotrema dennisii $\left(9^{\text {th }}\right)$, Steccherinum tenuispinum $\left(6^{\text {th }}\right)$, Stypella vermiformis $\left(4^{\text {th }}\right)$, Tomentella subclavigera $\left(6^{\text {th }}\right)$, Trechispora elongata $\left(2^{\text {nd }}\right)$, Trechispora laevis $\left(10^{\text {th }}\right)$, Trechispora silvae-ryae $\left(2^{\text {nd }}\right)$, Tremella polyporina $\left(5^{\text {th }}\right)$, Tubulicrinis hirtellus $\left(3^{\text {rd }}\right)$, Tulasnella albida $\left(7^{\text {th }}\right)$, Tyromyces fumidiceps $\left(4^{\text {th }}\right)$, and Xylodon nespori $\left(5^{\text {th }}\right)$.

In addition, 76 records are reported as new to some subzone of the boreal forest vegetation zone in Finland. The numbers of records new to subzones are: Hemiboreal, Aland (1a) 6; Hemiboreal, Oak Zone (1b) 10; Southern Boreal, Southwestern Finland and Southern Ostrobothnia (2a) 3; Middle Boreal, Ostrobothnia (3a) 11; Middle Boreal, Northern Carelia - Kainuu (3b) 21; Middle Boreal, Southwestern Lapland (3c) 15; Northern Boreal, North Ostrobothnia (4b) 6; Northern Boreal, Forest Lapland (4c) 4.

The species presented here divide into following red-listed categories: Endangered (5 species), Near Threatened (5), Data Deficient (4), Not Evaluated (3), Regionally Threatened (1), and Regionally Exctinct from subzone (1). If status of the species is Least Concern, it is not mentioned.

\section{List of species}

Species are listed in alphabetic order, regardless of their systematic position.

\section{Albatrellus citrinus Ryman}

Specimens examined: Nylandia, Helsinki, Keskuspuisto, Laakso, UCS 6677919:3384541, in eutrophic herb-rich forest, 15 Sep 2015, leg. KS A/150915 (K.S.); Satakunta, Pori, Saanteen Nasaret, UCS 6856:3211, in mixed herb-rich heath forest, Oct 2012, leg. KS.

These are the fourth and the fifth records in Finland. Previous records are from Finström (1a), Lohja (2a) and Helsinki, Pakila (1b) (Kotiranta et al. 2009, Savola 2012). Data Deficient.

Amylocorticiellum molle (Fr.) Spirin \& Zmitr.

Specimens examined: Ostrobottnia kajanensis, Sotkamo, Parkua, Känttäjä, UCS 7106357:3547381, on coniferous timber plank board (decay stage 3), in yard, 20 Aug 2008, PH 1673 (OULU), det. MK; and near the previous site UCS 7105860:3547390, on coniferous construction timber (diam. $7.5 \mathrm{~cm}$, decay stage 3) and on timber plank board (decay stage 2), in yard, 17 Sep 2011, leg. PH 2014, 2042 (OULU), det. MK; Sotkamo, Loso, Kuohunpuro, UCS 7105363:3546359, on cut log of Picea abies (decay stage 4), 29 Aug 2008, leg. PH 1681 (OULU), det. MK; Sotkamo, Korholanmäki, Losonvaara, UCS 7105447:3544939, on fallen trunk of Picea abies (decay stage 3), in old-growth mesic heath forest, 17 Oct 2011, leg. PH 2098 (OULU), det. MK.

New to Middle Boreal, Northern Carelia - Kainuu (3b).

Amylostereum areolatum (Chaillet ex Fr.) Boidin

Specimens examined: Ostrobottnia ouluensis, Oulu, Madekoski, Juurusoja, UCS 7204658:3436251, on fallen trunk of Picea abies (diam. $30 \mathrm{~cm}$, decay stage 3), in spruce mire, 27 Aug 2010, leg. MK 4/10 (OULU), det. MK \& O. Miettinen (specimen is sequenced); Oulu, Sanginjoki, Myllykoski, UCS 72154: 34427, on stump of Picea abies (diam. $40 \mathrm{~cm}$ ), in old-growth spruce mire, 30 Aug 2001, leg. MK 73/01 (OULU).

New to Middle Boreal, Ostrobothnia (3a). 
Anomoporia bombycina (Fr.) Pouzar

Specimen examined: Nylandia, Helsinki, Veräjämäki, UCS 6681379:3388471, on stump of Pinus sylvestris (diam. $30 \mathrm{~cm}$, decay stage 2), in rocky area with scarce forest, 23 Oct 2011, leg. KS $\mathrm{K} / 231011(\mathrm{H})$.

Re-found from Hemiboreal, Oak zone (1b). Near Threatened, Regionally Extinct from $1 \mathrm{~b}$.

Antrodia cretacea Runnel, Spirin \& Lõhmus

Specimen examined: Nylandia, Helsinki, Keskuspuisto, Paloheinä, UCS 6684907:3384781, on stump of Picea abies (diam. $40 \mathrm{~cm}$, decay stage 3), trunk was decayed by Fomitopsis pinicola, in eutrophic herb-rich forest with high amount of dead wood, 11 Oct. 2015, leg B. Weijola BWI/111015 (H), det. V. Spirin.

This is the third record in Finland. Previous records are from Helsinki (1b) and Sulkava (2b) (Savola 2015, Spirin et al. 2015).

Antrodia leucaena Y.C. Dai \& Niemelä

Specimens examined: Nylandia, Hyvinkää, Sveitsin puisto Nature Reserve, UCS 6727232:3381295, on stump of Alnus incana (diam. $20 \mathrm{~cm}$, decay stage 3), in moist, mixed herbrich forest, 29 Sep 2015, leg. KS K/290915 (H), det. V. Spirin; Savonia australis, Hirvensalmi, Haukonmäki, UCS 6835590:3487960, on decorticated Populus tremula (diam. $20 \mathrm{~cm}$, decay stage 2), 28 Apr 2016, leg. J. Uimonen (H), det. HK, conf. V. Spirin.

New to Southern boreal, SW Finland and Southern Ostrobothnia (2a). These are the the third and the fourth localities in Finland. Previous records are from Helsinki (1b) and Savonranta (2b), and substrata were Populus tremula (Spirin et al. 2013 and collections of herbarium H).

Antrodia mappa (Overh. \& J. Lowe) Miettinen \& Vlasák

Specimen examined: Ostrobottnia kajanensis, Puolanka, Kettukallio, UCS 7185595:3524988, on fallen trunk (conifer?), in old-growth mesic heath forest, 26 Aug 2015, leg. M. Tyyskä \& P. Keihäs (OULU), det. MK.

New to Middle Boreal, Ostrobothnia (3a). This is the fifth record in Finland. Previous records are from Nauvo ( $1 \mathrm{~b}$, on Pinus sylvestris), Korpilahti ( $2 \mathrm{~b}$, on deciduous tree), Savonranta (2b, on Populus tremula), and Kankaanpää (3a, on Salix caprea), (Kotiranta et al. 2009, Kunttu et al. 2011). Endangered.

Antrodia piceata Runnel, Spirin \& Vlasák ("Antrodia sitchensis" (D.V. Baxter) Gilb. \& Ryvarden ss. auct.)

Specimens examined: Nylandia, Raasepori, Rödjeberget, UCS 6676367: 3311007, on fallen trunks of Picea abies (diam. $40 \mathrm{~cm}$, decay stage 4 and diam. $40 \mathrm{~cm}$, decay stage 3), in sprucedominated mesic heath forest, 4 Oct 2015, leg. JP 3016, 3024 (H, J.P.); Nylandia, Helsinki, Keskuspuisto, Paloheinä, UCS 6684830:3385269, on stump of Picea abies (diam. $45 \mathrm{~cm}$, decay stage 3, with Fomitopsis pinicola), in herb-rich forest with high amount of dead wood, 27 Sep 2015, leg. KS E/270915 (H), det. V. Spirin; Tavastia borealis, Keuruu, Hirvo, Päijänteenmäki, UCS 6903019:3388373, on fallen broken, partly debarked trunk of Picea abies (diam. $18 \mathrm{~cm}$, decay stage 3), near spruce mire in spruce-dominated mesic heath forest, 1 Sep 2015, leg. M. Kokkonen 5 (JYV).

Recent research (Spirin et al. 2015) has shown, that the American A. sitchensis has a European counter part, A. piceata. The previous Finnish records of A. sitchensis thus belong most probably to A. piceata, but so far only some specimens have been confirmed, like specimens from two localities in Helsinki (1b), Padasjoki (2a) and Ruovesi (2b) (Savola 2012, Spirin et al. 2015). Additionally, there are 11 previous records known - identified as A. sitchensis - throughout Finland (Kotiranta et al. 2009, Kunttu et al. 2014, 2015). Endangered. 
Antrodiella ichnusana Berniccia, Renv. \& Arras

Specimen examined: Alandia, Finström, Prästgårdnäset Nature Reserve, UCS 6703689:3109402, on dead standing branch of Corylus avellana (diam. $4 \mathrm{~cm}$, decay stage 2), in herb-rich forest/wooded pasture, 24 Sep 2015, leg. JP 2992 (H, J.P.) conf. O. Miettinen.

New to Hemiboreal, Åland (1a).

Athelia subovata Jülich \& Hjortstam

Specimen examined: Ostrobottnia kajanensis, Puolanka, Paljakka, Mustakumpu UCS 7172617:3550607, on piece of Picea abies trunk (diam. $15 \mathrm{~cm}$, decay stage 3), in old-growth mesic heath forest, 30 Sep 2011, leg. PH 2052 (OULU), det. MK; Sotkamo, Korholanmäki, Losonvaara, UCS 7106009:3544938, on fallen trunk of Populus tremula (diam. $100 \mathrm{~cm}$, decay stage 3), in oldgrowth spruce mire, 20 Oct 2011, PH 2124 (OULU), det. MK.

New to Middle boreal, Northern Carelia - Kainuu (3b).

Byssocorticium pulchrum (S. Lundell) M.P. Christ.

Specimen examined: Ostrobottnia ultima, Rovaniemi, Pisavaara Strict Nature Reserve, Teeripäri, UCS 7358037:3414457, on stump of Pinus sylvestris (diam.25 cm, decay stage 2), in old-growth xeric heath forest, 3 Sep 2015, leg. MK 95/15 (OULU).

New to Middle Boreal, Southwestern Lapland (3c).

Byssoporia mollicula (Bourdot) Larsen \& Zak

Specimen examined: Nylandia, Helsinki, Santahamina, Kissalampi, UCS 6672222:3392778, on cut $\log$ (diam. $25 \mathrm{~cm}$, decay stage 3), in herb-rich forest, 26 Nov 2011, leg. KS (H), det. J. Kinnunen.

New to Hemiboreal, Oak zone (1b).

\section{Ceraceomyces microsporus K.H. Larsson}

Specimen examined: Lapponia sompiensis, Sodankylä, Pomokaira, Jäkälävaara, UCS 7552505:3468330, on cut log of Pinus sylvestris (diam. 34, decay stage 4, kelo tree), 25 Aug 2009, leg. PK 5121 (TUR), det. JP.

New to Northern Boreal, North Ostrobothnia (4b).

Ceriporia aurantiocarnescens (Henn.) M. Pieri \& B. Rivoire

Specimens examined: Nylandia, Helsinki, Hallainvuori, UCS 6680989:3391741, on stump of Betula pendula (diam. $30 \mathrm{~cm}$, decay stage 3), in old-growth heath forest with high amount of dead wood, 1 Oct 2014, leg. KS W/011014 (H), det. V. Spirin; Helsinki, Niinisaari, UCS 6680840:3399215, on stump of Salix caprea (diam. $15 \mathrm{~cm}$ ), in mesic heath forest with a high amount of dead wood, 5 Oct. 2014, leg. B. Weijola, BW12/051014 (H), det. V. Spirin.

These are the second and the third record in Finland. The first record is from Helsinki, Mustavuori (1b) according to collections of herbarium $\mathrm{H}$.

Ceriporia bresadolae (Bourdot \& Galzin) Bondartsev \& Singer

Specimens examined: Ostrobottnia ouluensis, Muhos, Laitasaari, Rova, UCS 719759:344645, on timber plank board, Pinus sylvestris, 10 Oct 2005, MK 119/05 (OULU); Ostrobottnia ouluensis; Oulu, Välivainio, Susitie, UCS 7215933:3428369, on decorticated timber, Pinus sylvestris (diam. 7 $\mathrm{cm}$, decay stage 1), in garden, 23 Sep 2014, leg. T. Kallio 1450 (H), det. T. Niemelä; Lapponia inarensis, Inari, Lemmenjoki National Park, close to Ravadasköngäs, UCS 762:345, on decorticated, hard Pinus sylvestris on ground near shore in pine-dominated old-growth forest, 1 Sep 1982, HK 3988 (H, H.K.), det. V. Spirin.

New to Finland. New to Middle boreal, Ostrobothnia (3a) and Northern Boreal, Forest Lapland (4c). 
Chaetoporellus curvisporus (J. Erikss. \& Hjortstam) J. Erikss. \& Hjortstam

Specimen examined: Ostrobottnia media, Pyhäjärvi, Jokikylä, Varpusuo, UCS 7072532:3449950, on stump of Picea abies (diam. $16 \mathrm{~cm}$, decay stage 3), in spruce mire, 9 Sep 2015, leg. MK 31/15 (OULU).

This is the seventh record in Finland. Previous records are from Koski (2a), Lammi (2a), Padasjoki (2a), Muurame (2b), Saarijärvi (3a) and Paltamo (3b) (on Pinus, Picea and Betula) (Kotiranta et al. 2009, Kunttu et al. 2011, 2015). Near Threatened.

Clavaria amoenoides Corner, K.S. Thind \& Anand

Fig. 2

Specimen examined: Ostrobottnia ultima, Kemijärvi, Raajärvi, UCS 738575:349637, in dry grassland with Antennaria dioica, 9 Aug 2015, leg. \& det. TK 1859 (BRA), conf. I. Kautmanova.

New to Northern boreal, North Ostrobothnia (4b).

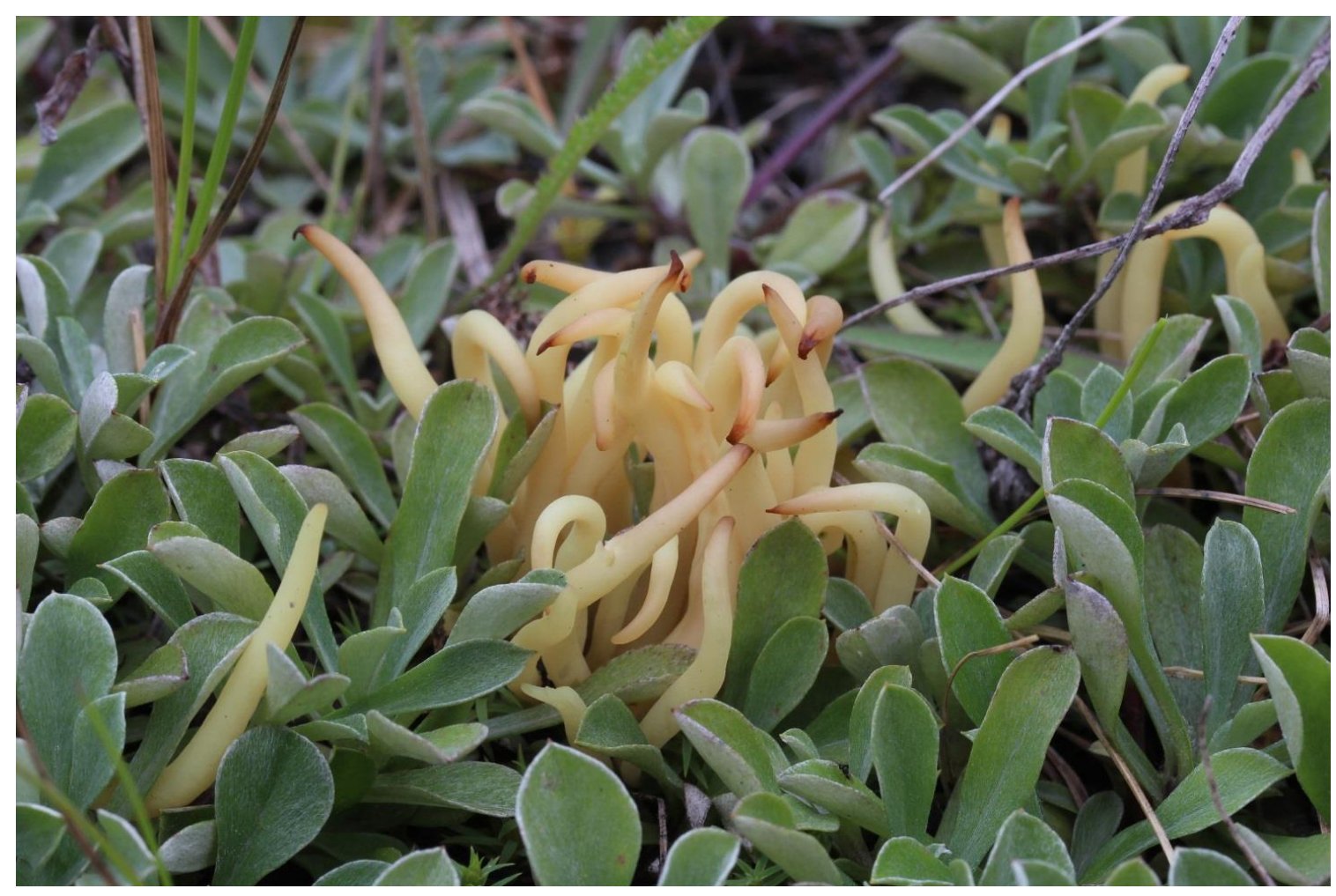

Fig. 2 - Clavaria amoenoides in Kemijärvi (TK 1859). Photo: Tapio Kekki.

Clavaria flavipes Pers.

Fig. 3

Specimens examined: Tavastia australis, Juupajoki, Hyytiälä, UCS 6862:3357, in unfertilized field among deep grass, 6 Sep 2005, leg. S. Jakobsson 4472, S. Adamčík \& I. Kautmanová (H); Ostrobottnia kajanensis, Kajaani, city centre, Seminaari, UCS 71257:35351, in dry grassland with Sorbus, 25 Sep 2015, leg. TH 150005 (OULU); Kajaani, Vimpelinlaakso, UCS 71240:35349, in dry grassland, 23 Sep 2015, leg. TH 150006 (OULU); Ostrobottnia kajanensis, Sotkamo, Rikkola, Känttäjä, UCS 71063:35474, in dry grassland, 21 Sep 2015, leg. TH 150007 (OULU); Ostrobottnia ultima, Tornio, Arpela, UCS 732959:338226, on grazing ground for sheep, 26 Sep 2015, leg. TK 2137 (OULU); Ostrobottnia ultima, Keminmaa, old church area, UCS 730413:338800, in calciferous grassland, 27 Sep 2015, leg. \& det TK 2150 (BRA), conf. I. Kautmanova; Ostrobottnia ultima, Rovaniemi, Kiiruna, UCS 737878:344484, in dry grassland with Pinus, 21 Sep 2015, leg. TK 2093 (OULU); Rovaniemi, Muurola, UCS 73657:34273, in dry grassland with Pinus, 28 Sep 2015, leg. \& det TK 2185 (BRA), conf. I. Kautmanova.

New to Middle boreal, Northern Carelia - Kainuu (3b), Middle boreal, Southwestern Lapland (3c) and Northern boreal, North Ostrobothnia (4b). There are two previous records from Southern boreal zone: Sipoo (2a) and Saari (2b) (Kotiranta et al 2009). Not Evaluated. 


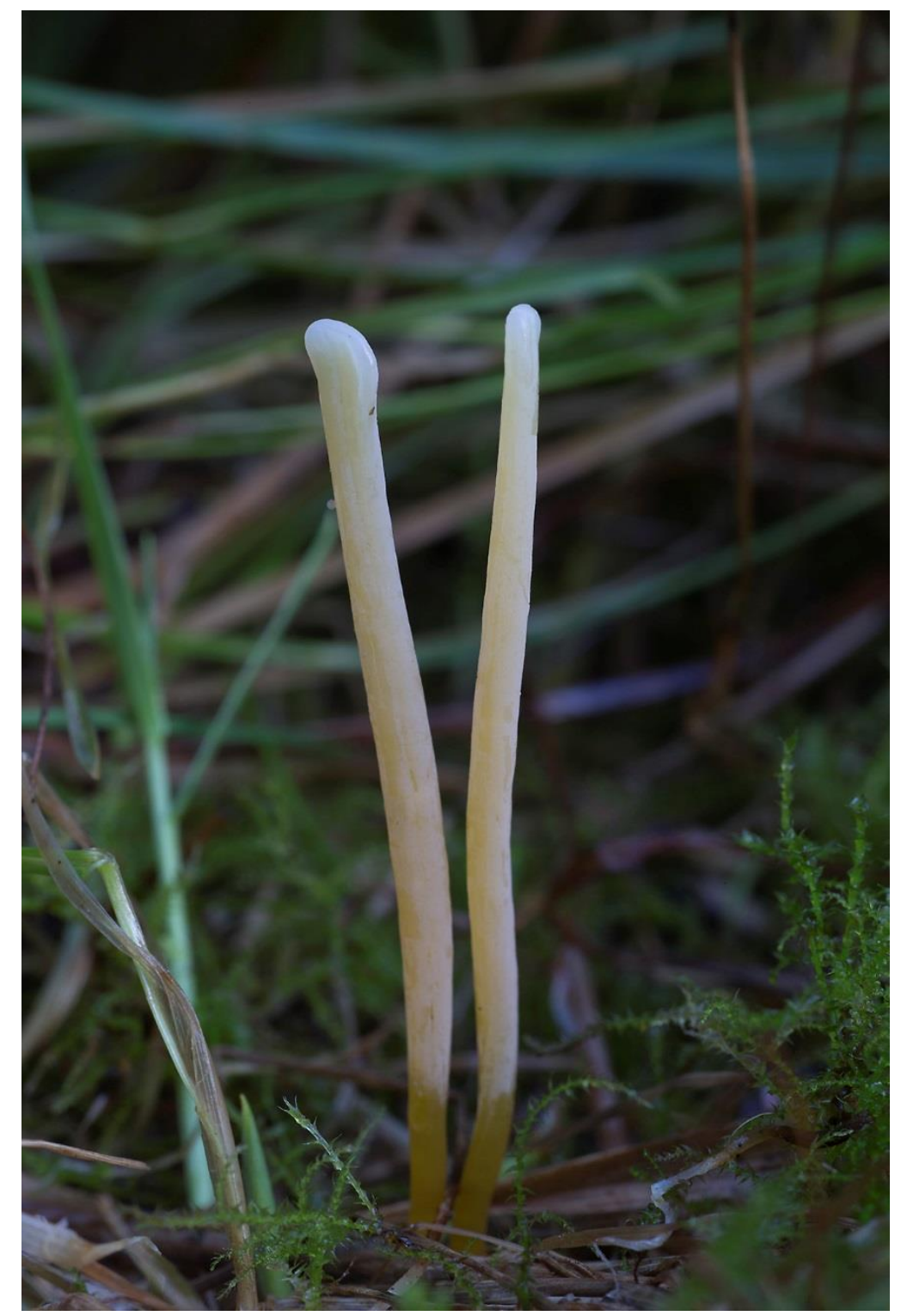

Fig. 3 - Clavaria flavipes in Sotkamo (TH 150007). Photo: Teppo Helo.

Clavaria greletii Boud.

Fig. 4

Specimens examined: Ostrobottnia kajanensis, Kajaani, Teppana, UCS 71261:35362, on lawn, 6 Aug 2015, leg. TH 150001 (OULU); Kajaani, Kaukametsä, UCS 71253:35361, in grassland next to road, 4 Oct 2015, leg. TH 150003 (OULU); Ostrobottnia kajanensis, Sotkamo, church, UCS 71153:35685, in dry grassland, 8 Aug 2015, leg. TH 150002 (OULU).

New to Middle boreal, Northern Carelia - Kainuu (3b). There are five previous records from Finland: Ikaalinen (2a), Pälkäne (2a), Lappeenranta (2b), Pieksämäki (2b), and Keminmaa (3c) (Kotiranta et al. 2009).

\section{Clavaria rosea Dalman}

Specimens examined: Ostrobottnia kajanensis, Kajaani, city center, church, UCS 71252:35354, on lawn with Picea abies, 8 Aug 2015, TH 150008 (OULU); Ostrobottnia ultima, Rovaniemi, Kiiruna, UCS 737876:344484, in dry and sandy grassland under Pinus sylvestris, 6 Aug 2015, TK 1829 (OULU).

New to Middle boreal, Northern Carelia - Kainuu (3b) and Northern boreal, North Ostrobothnia (4b). Previously this species was considered as rare but currently 11 records are known from different parts of Finland (Kunttu et al 2014, Kekki 2015). 


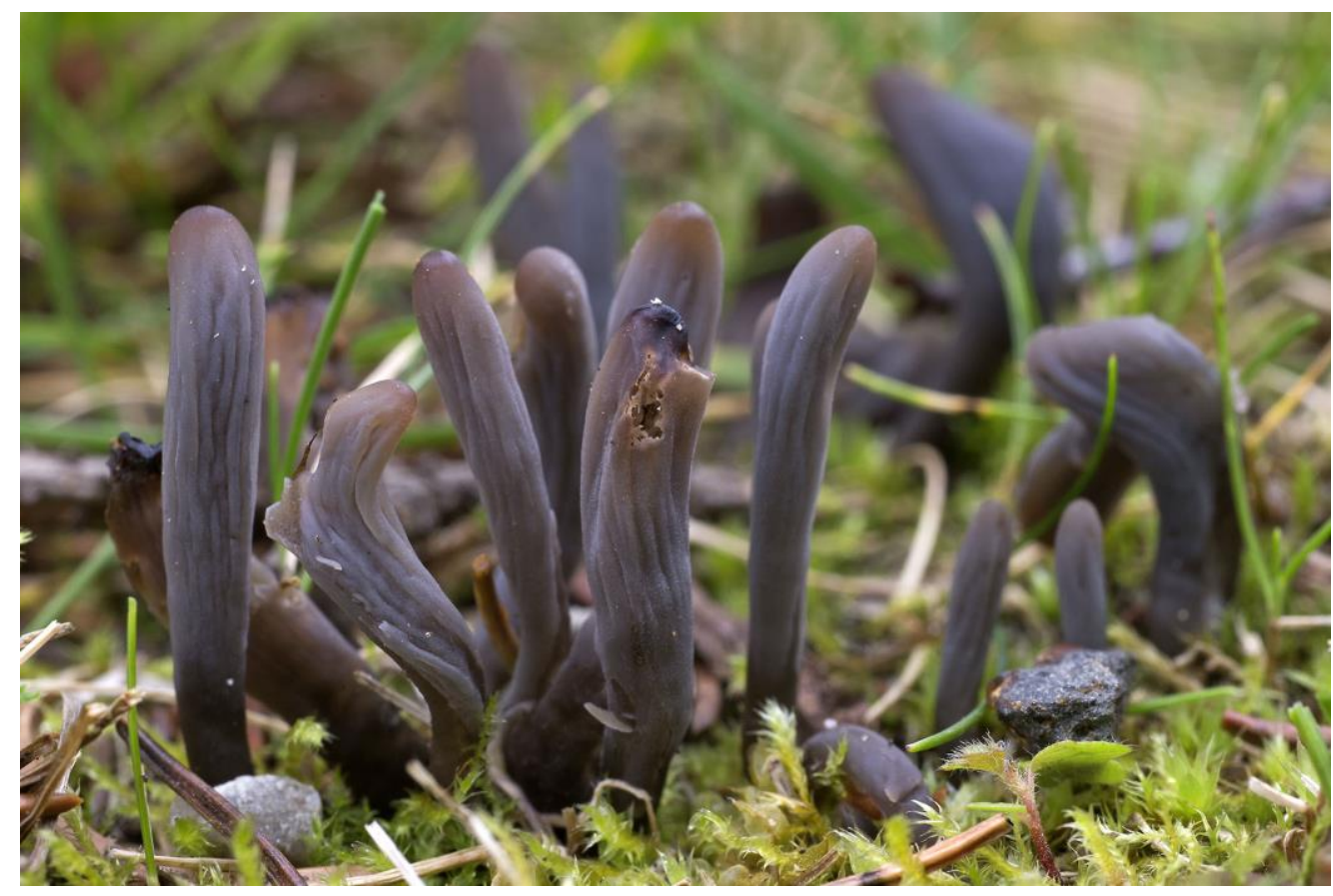

Fig. 4 - Clavaria greletii in Kajaani (TH 150001). Photo: Teppo Helo.

Clavaria tenuipes Berk. \& Broome ss Corner non Schild

Fig. 5

Specimens examined: Savonia borealis, Pieksämäki, Ankele, UCS 6885215:3521280, in calciferous grassland at road shoulder, 5 Sep 2014, leg. \& det. TK 1576 (BRA), conf. I. Kautmanova; Ostrobottnia ultima, Rovaniemi, Kiiruna, UCS 737876:344484, on sandy ground in a yard with planted mountain plants, 10 Aug 2010, leg. TK 32 (TUR) and same place in 13 Aug 2015, leg. \& det. TK 1871 (BRA), conf. I. Kautmanova; Rovaniemi, Katajaranta, UCS 737897:344453, in grassland with Pinus and Syringa, 21 Sep 2015, leg. TK 2096 (OULU).

New to Finland. New to Southern boreal, Lake district (2a) and Northern boreal, North Ostrobothnia (4b).

\section{Clavulinopsis helvola (Pers.) Corner}

Specimens examined: Alandia, Lemland, Nåtö Nature Reserve, UCS 6680439:3109521, on ground in pollard meadow, 22 Sep 2015, leg. JP 2983 (H, J.P.); Ostrobottnia kajanensis, Sotkamo cemetery, UCS 71148:35686, on lawn with Pinus sylvestris and Betula sp., 30 Sep 2015, leg. TH 150004 (OULU).

New to Hemiboreal, Åland (1a) and Middle boreal, Northern Carelia - Kainuu (3b).

Colacogloea peniophorae (Bourdot \& Galzin) Oberw., R. Bauer \& Bandoni

Specimens examined: Regio aboënsis, Kemiönsaari, Örö, west shore, UCS 6642:3237, on conifer drift wood log (diam. $24 \mathrm{~cm}$, decay stage 4, length $5.3 \mathrm{~m}$ ); on drift wood log of Betula sp. (diam. $14 \mathrm{~cm}$, decay stage 3, length $3.2 \mathrm{~m}$ ); on unidentified drift wood log (diam. $18 \mathrm{~cm}$, decay stage 2, length $4 \mathrm{~m}$ ), on sand beach, 2 Oct 2015, leg. PK 8834, 8841, 8860 (TUR), det. MK.

There are nine previous records made in Finland. All records are from the southwestern archipelago or the south coast (subzones 1a and 1b) (Kotiranta et al. 2009, Kunttu et al. 2009, 2012, 2015, Miettinen 2012).

Coniophora fusispora (Cooke \& Ellis) Sacc.

Specimen examined: Ostrobottnia ultima, Tervola, Pisavaara Strict Nature Reserve, Pihlajakurunlampi, UCS 7353851:3412927, on fallen trunk of Picea abies (diam. $11 \mathrm{~cm}$, decay stage 3), in old-growth mesic heath forest, 4 Sep 2015, leg. MK 11/15 (OULU).

New to Middle Boreal, Southwestern Lapland (3c). 


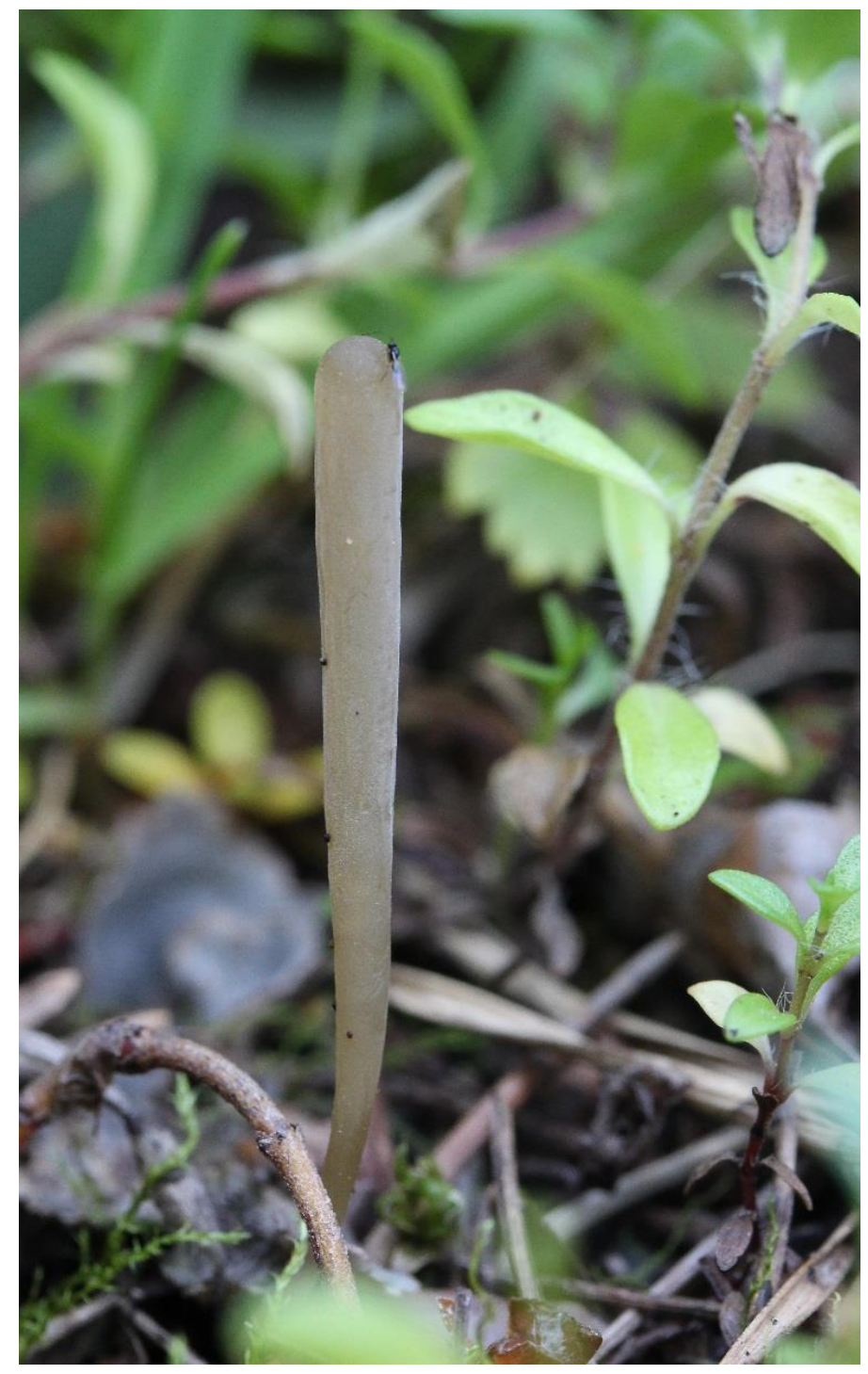

Fig. 5 - Clavaria tenuipes in Rovaniemi (TK 1871). Photo: Tapio Kekki.

\section{Crustomyces subabruptus (Bourdot \& Galzin) Jülich}

Specimen examined: Regio aboënsis, Kemiönsaari, Örö, west shore, UCS 6641:3237, on drift wood $\log$ (diam. 33 cm, decay stage 1), on sand beach, 4 Oct 2015, leg. PK 9171b (TUR), det. HK.

This is the second record in Finland. The first record was made in Parainen (1b) where basidiome was found on corticated Tilia cordata (Kotiranta et al. 2009). Data Deficient.

\section{Cystostereum murraii (Berk. \& M.A. Curtis) Pouzar}

Specimen examined: Alandia, Lemland, Harskata Nature Reserve, UCS 6679169: 3108785, on fallen trunk of Picea abies (diam. $20 \mathrm{~cm}$, decay stage 2), in herb-rich heath forest, 23 Sep 2015, leg. JP 2987 (H, J.P.). Finland.

New to Hemiboreal, Åland (1a). Near Threatened and Regionally Threatened in Southern

\section{Gloeophyllum carbonarium (Berk. \& M.A. Curtis) Ryvarden}

Specimens examined: Ostrobottnia kajanensis, Paltamo, Kuuskanlahti, Teerivaara, UCS 7148496:3513242, on charred fallen trunk and rootplate of Pinus sylvestris (diam. $35 \mathrm{~cm}$, decay stage 2), in old-growth xeric heath forest, 21 Oct 2012 leg. PH 2461 (OULU), conf. TH \& MK; Ostrobottnia kajanensis, Kajaani, Vuolijoki, Kokkosalo, UCS 7111214:3494561, on charred Pinus sylvestris (diam. 40 cm, decay stage 4), Mkk, 16 Sep 2013, leg. PH 2493 (OULU), conf.TH \& MK. 
New to Middle boreal, Ostrobothnia (3a). These are the ninth and the tenth records in Finland. Previous records are from Lapland, Central and Eastern Finland (Kotiranta et al. 2009, Kunttu et al. 2014). Endangered.

Hyphoderma definitum (H.S. Jacks.) Donk

Specimen examined: Ostrobottnia kajanensis, Sotkamo, Parkua, Känttäjä, UCS 7106356:3547390, on construction timber, (diam. 18 cm, decay stage 4), in yard, 29 Aug 2008, leg. PH 1677 (OULU), det. MK.

New to Middle Boreal, Northern Carelia - Kainuu (3b).

Hypochnicium albostramineum (Bres.) Hallenb.

Specimen examined: Ostrobottnia ultima, Rovaniemi, Pisavaara Strict Nature Reserve, Teeripäri, UCS 7358602:3414704, on coniferous stump (diam. $35 \mathrm{~cm}$, decay stage 1), in oldgrowth mesic heath forest, 3 Oct 2015, leg. MK 93/15 (OULU).

New to Middle Boreal, Southwestern Lapland (3c).

Jaapia ochroleuca (Bres.) Nannf. \& J. Erikss.

Specimen examined: Ostrobottnia media, Pyhäjärvi, Jokikylä, Varpusuo, UCS 7072547:3449942, fallen trunk of conifer (diam. $10 \mathrm{~cm}$, decay stage 2), in spruce mire, 9 Sep 2015, leg. MK 42b/15 (OULU).

New to Middle Boreal, Ostrobothnia (3a).

Lagarobasidium detriticum (Bourdot \& Galzin) Jülich

Specimen examined: Ostrobottnia kajanensis, Kajaani, Variskangas, Tilhitie, UCS 7123737:3537548, on fallen trunk of Betula sp. (diam. $3 \mathrm{~cm}$, decay stage 3), in moist, fairly young birch dominated forest, 1 Nov 2011, PH 2190 (OULU), det. MK.

New to Middle Boreal, Northern Carelia - Kainuu (3b).

Lentaria byssiseda (Pers. : Fr.) Corner

Specimen examined: Alandia, Finström, Prästgårdnäset Nature Reserve, UCS 6679169:3108785, on fallen broken trunk of Quercus robur (diam. $50 \mathrm{~cm}$, decay stage 3), in herbrich forest and wooded pasture, 24 Sep 2015, leg. JP 2993 (H, J.P.).

New to Hemiboreal, Åland (1a).

\section{Leucogyrophana mollusca (Fr.) Pouzar}

Specimen examined: Ostrobottnia ouluensis, Muhos, Laitasaari, Rova UCS 71975:34464, on timber plank board, in yard, 18 Sep 2015, MK 80/15 (OULU).

New to Middle Boreal, Ostrobothnia (3a).

Leptosporomyces galzinii (Bourdot) Jülich

Specimen examined: Ostrobottnia ultima, Rovaniemi, Pisavaara Strict Nature Reserve, UCS 7359172:3416115, on fallen trunk of Picea abies (diam. $20 \mathrm{~cm}$, decay stage 3), in spruce mire, 3 Oct 2015, leg. MK 84/15 (OULU).

New to Middle Boreal, Southwestern Lapland (3c).

Luellia recondita (H.S. Jacks.) K.H. Larsson \& Hjortstam

Specimen examined: Ostrobottnia ultima, Rovaniemi, Pisavaara Strict Nature Reserve, UCS 7359421:3416585, on fallen trunk of Picea abies (diam. $6 \mathrm{~cm}$, decay stage 3), in old-growth mesic heath forest, 17 Sep 2015, leg. MK 67b/15 \& PH (OULU), det. MK. This specimen was varia furcata.

New to Middle Boreal, Southwestern Lapland (3c). This is the sixth record in Finland. Previous records are from Eckerö (1a), Kemiönsaari (1b), Tammisaari (1b), Helsinki (1b), and Hamina (2a), where basidiomata grew on Picea abies and Juniperus communis (Kotiranta et al. 2009, Kunttu et al. 2012, 2015). 
Oligoporus romellii (Pieri \& Rivoire) Niemelä

Specimens examined: Regio aboënsis, Kemiönsaari, Örö, west shore, UCS 6642:3237, on drift wood log of Picea abies (diam. $21 \mathrm{~cm}$, decay stage 3), on coniferous drift wood log (diam. 18 cm, decay stage 4), on sand beach, 2 Oct 2015, leg. PK 8867, 8891 (TUR), det. MK; Nylandia, Helsinki, Laajasalo, Alustalaismäki, UCS 6675323:3392641, on stump of Pinus sylvestris (diam. 18 cm, decay stage 3), in forested rocky area, 18 Oct 2014, leg. KS S/181014, (H), det. V. Spirin; Specimens examined: Nylandia, Hyvinkää, Sveitsin puisto Nature Reserve, UCS 67827232:3381295, on stump of Pinus sylvestris (diam. $17 \mathrm{~cm}$ ), in xeric old-growth pine forest, 20 Sep 2015, leg. KS E/200915 (H), det. V. Spirin; Ostrobottnia kajanensis, Sotkamo, Rikkola, Känttäjä, UCS 7106337:3547384, on coniferous construction timber (diam. $18 \mathrm{~cm}$, decay stage 3), in yard, 8 Nov 2011, PH 2231a (OULU), det. MK; Ostrobottnia ultima, Rovaniemi, Pisavaara Strict Nature Reserve, UCS 7359572:3416555, on fallen Picea abies (diam. $35 \mathrm{~cm}$, decay stage 1), in oldgrowth mesic heath forest, 25 Sep 2011, MK 67/11 (OULU), det. MK \& O. Miettinen (specimen is sequenced) and on same trunk: 17 Sep 2015, MK 50/15 \& PH (OULU), det. MK.

New to Hemiboreal, Oak zone (1b), Southern Boreal, SW Finland and Southern Ostrobothnia (2a) and Middle Boreal, Southwestern Lapland (3c). There are 12 previous record from Finland: in Helsinki 10 localities (1a), Lammi (2a), and Ilomantsi (3b) (Kotiranta et al. 2009, Niemelä 2012, Savola 2015). Not Evaluated.

Paullicorticium pearsonii (Bourdot) J. Erikss.

Specimen examined: Regio aboënsis, Kemiönsaari, Dragsfjärd, Örö, west shore, UCS 6642:3237, on conifer log, drift wood, (diam. $24 \mathrm{~cm}$, decay stage 4, length $5.3 \mathrm{~m}$ ), on sand beach, 2 Oct 2015, leg. PK 8833, 8834 (TUR), det. MK.

This is the fifth record in Finland. Previous are from two sites in Helsinki (1b) and two sites in Inkoo (1b). There basidiomata grew on coniferous timber and logs of Pinus sylvestris (Kotiranta et al. 2009).

Phanerochaete livescens (P. Karst.) Volobuev \& Spirin

Specimen examined: Nylandia, Helsinki, Haltiala, UCS 6685308:3384956, on stump of Alnus incana (diam. $25 \mathrm{~cm}$, decay stage 3), in herb-rich spruce mire with high amount of dead wood, 17 Oct 2015, leg. KS, D/171015 (H), det.V. Spirin.

New to Hemiboreal (1b). This is the third record in Finland. Previous records are from Tammela (2a) on Tilia cordata and Ilomantsi (2b) on Populus tremula (Karsten 1895, Volobuev et al. 2015).

Phlebia subcretacea (Litsch.) M.P. Christ.

Specimen examined: Savukoski, Värriö Strict Nature Reserve, Jyppyrävaara, UCS 7521467:3609246, whole fallen trunk of Pinus sylvestris (diam. 24, decay stage 2, kelo tree), 22 Sep 2009, leg. PK 5658 (TUR), det. JP.

New to Northern Boreal, Forest Lapland (4c). This is the ninth record in Finland. Previous records derive throughout Finland (Kotiranta et al. 2009, Kunttu et al. 2015). Species is found mainly on large trunks of Pinus sylvestris.

Phlebiella christiansenii (Parmasto) K.H. Larss. \& Hjortstam

Specimen examined: Ostrobottnia kajanensis, Paltamo, Melalahti, Keräsenvaara, UCS 7151386:3528561, on top of fallen Picea abies trunk (diam. $7 \mathrm{~cm}$, decay stage 3), in old-growth xeric heath forest, 15 Sep 2009, leg. PH 1843 (OULU) det. MK.

New to Middle Boreal, Northern Carelia - Kainuu (3b).

Postia hibernica (Berk. \& Broome) Jülich

Specimen examined: Lapponia inarensis, Inari, Matojärvi, UCS 76564:35064, on cut $\log$ of Pinus sylvestris (diam. 15 cm), in heath forest, 17 Oct 1999, leg. MK 264/99 (OULU). 
New to Northern Boreal, Forest Lapland (4c).

\section{Postia lateritia Renvall}

Specimens examined: Regio aboënsis, Kemiönsaari, Dragsfjärd, Stora Buskär, UCS 6644:3229, on unidentified drift wood $\log$ (diam. $14 \mathrm{~cm}$, decay stage 2, length $2.9 \mathrm{~m}$ ), on stony shore, 2 Sep 2015, leg. PK 8694 (TUR), det. MK; Kemiönsaari, Örö, Storviken, UCS 6642:3238, on fallen trunk of Pinus sylvestris, in pine-dominated mesic heath forest, 5 Oct 2015, leg. PK 9230 (TUR), det. MK.

New to Hemiboreal, Oak zone (1b). Near Threatened.

\section{Pseudotomentella mucidula (P. Karst.) Svrček}

Specimen examined: Ostrobottnia kajanensis, Sotkamo, Saukkoperä, Niemilehto, UCS 7084379:3590957, on fallen trunk of Pinus sylvestris (diam. $65 \mathrm{~cm}$, decay stage 3), in old-growth mesic heath forest, 1 Sep 2008, leg. PH 1714 (OULU), det. MK.

New to Middle Boreal, Northern Carelia - Kainuu (3b).

\section{Pycnoporellus alboluteus (Ellis \& Everhart) Kotl. \& Pouzar}

Fig. 6.

Specimens examined: Ostrobottnia kajanensis, Puolanka, Paljakka, Latvavaara Nature Reserve, UCS 71787-90:35467-9, nine fallen, partly decorticated trunks of Picea abies decayed by Fomitopsis pinicola (Sw.) P. Karst (diam. range 22-45 cm (md $30 \mathrm{~cm}$ ), decay stage range 1-2 (md 1), in old-growth spruce forest, 28 Oct 2015 \& 2 Nov 2015, TH 150013-150021 (OULU).

New to Middle Boreal, Northern Carelia - Kainuu (3b). This is the eigth locality in Finland. Previous localities are in Tervola (3c), Rovaniemi (3c), Pudasjärvi (4a), Taivalkoski (4a), and Kemijärvi (4b), Rovaniemi (4b) (Kotiranta et al. 2009, Kunttu et al. 2015). The number of occupied trunks is much higher. Substrata: Picea abies, Alnus incana and Populus tremula, almost solely on trees decayed by Fomitopsis pinicola. Endangered.

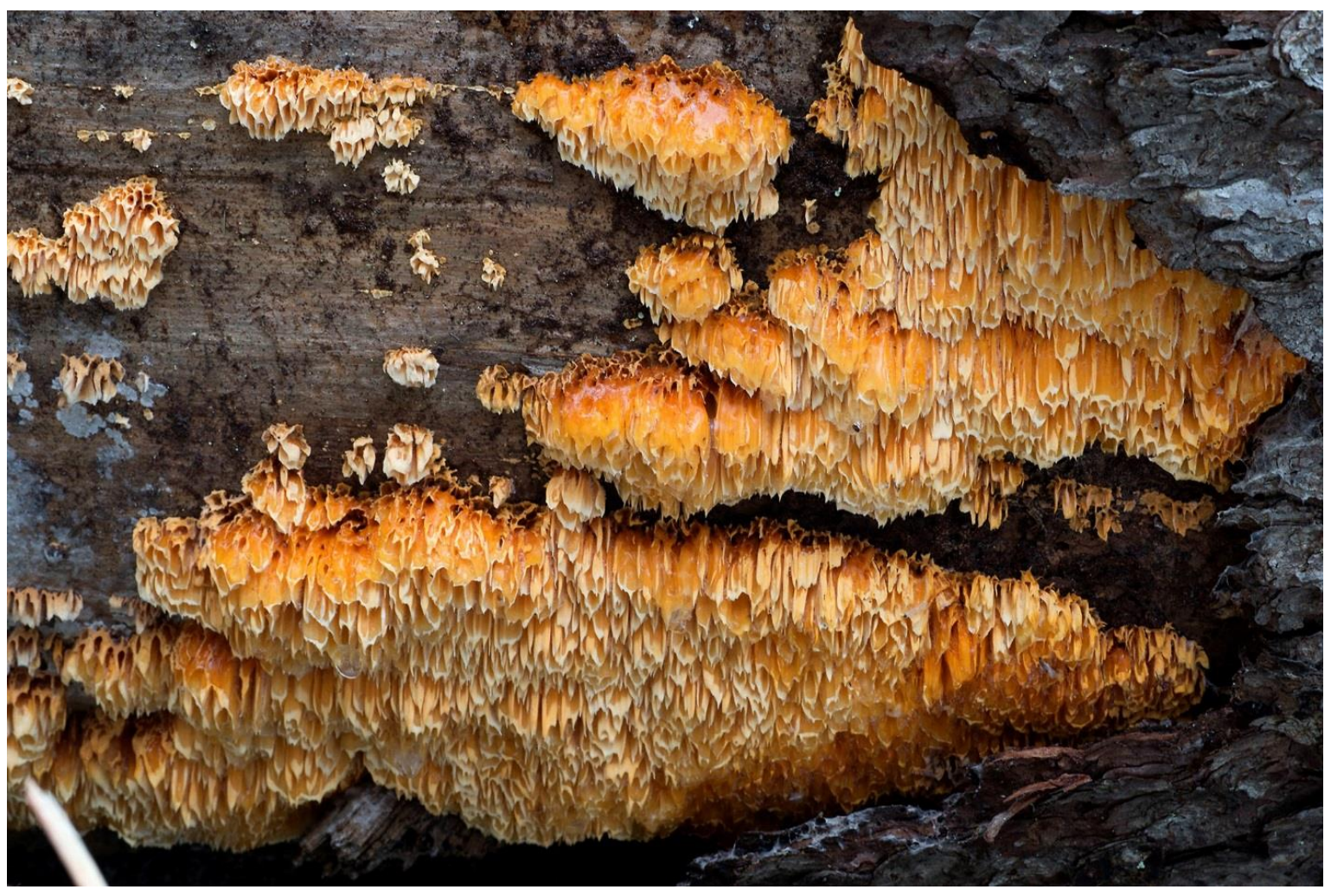

Fig. 6 - Pycnoporellus alboluteus on fallen trunk of Picea abies in Puolanka (TH 150013). Photo: Teppo Helo. 
Pycnoporellus fulgens (Fr.) Donk

Specimen examined: Alandia, Eckerö, Skeppsvik, UCS 6698957:3087797, on cut stump of Picea abies (diam. $45 \mathrm{~cm}$, decay stage 4), in spruce-dominated herb-rich heath forest, 3 Jun 2015, leg. PK 8670 (TUR).

New to Hemiboreal, Åland (1a).

\section{Ramariopsis kunzei (Fr.) Corner}

Specimen examined: Ostrobottnia ultima, Rovaniemi, Kiiruna, UCS 737876:344484, in grassland at sandy road shoulder, 10 Aug 2015, leg. TK 1863 (OULU).

New to Northern boreal, North Ostrobothnia (4b).

\section{Ramariopsis tenuiramosa Corner}

Specimen examined: Ostrobottnia kajanensis, Sotkamo, Rikkola, Känttäjä, UCS 71063:35473, under Rubus idaeus, 21 Sep 2015, leg. PH 2805 (OULU) det. TH.

New to Middle boreal, Northern Carelia - Kainuu (3b).

\section{Renatobasidium notabile Hauerslev}

Specimen examined: Nylandia, Inkoo, Älgsjö (Elisaari), UCS 66562:33275, on corticated Juniperus communis, (diam. $1.1 \mathrm{~cm}$, decay stage 1), in herb-rich forest, 23 Sep 2004, leg. HK 27046 (H.K.).

New to Finland. New to Hemiboreal, Oak zone (1b).

\section{Resinicium pinicola (J. Erikss.) J. Eriksson \& Hjortstam}

Specimens examined: Regio aboënsis, Kemiönsaari, Örö, west shore, UCS 6641:3237, on three substrata: on drift wood log of Picea abies (diam. $13 \mathrm{~cm}$, decay stage 1), drift wood $\log$ (diam. $22 \mathrm{~cm}$, decay stage 3), drift wood $\log$ (diam. $30 \mathrm{~cm}$, decay stage 2), on sand beach, 3 Oct 2015, leg. PK 8943, 9001, 9041 (TUR), det. MK, conf. HK.

There are three earlier collections of this species: two sites in Parainen (1b) and one earlier from Örö island (1b), i.e. same site as these two new records. Basidiomata were found on Pinus sylvestris in all cases (Kotiranta et al. 2009, Kunttu et al. 2014).

\section{Sarcoporia polyspora $\mathrm{P}$. Karst.}

Specimen examined: Regio aboënsis, Kemiönsaari, Örö, west shore, UCS 6641:3237, on coniferous drift wood log (diam. $32 \mathrm{~cm}$, decay stage 2), on sand beach, 3 Oct 2015, leg. PK 8991 (TUR), det. MK.

New to Hemiboreal, Oak zone (1b).

\section{Scytinostroma praestans (H.S. Jacks.) Donk}

Specimen examined: Ostrobottnia ultima, Rovaniemi, Pisavaara Strict Nature Reserve, UCS 7359572:3416555, fallen trunk of Picea abies (diam. $35 \mathrm{~cm}$, decay stage 2), in old-growth mesic heath forest, 17 Sep 2015 MK 50/15 \& PH (OULU), det. MK.

New to Middle Boreal, Southwestern Lapland (3c).

\section{Scytinostromella nannfeldtii (J. Erikss.) G.W. Freeman \& R.H. Petersen}

Specimen examined: Ostrobottnia kajanensis, Hyrynsalmi, Lietekylä, Peuravaara, UCS 7173896:3557320, on stump of Betula sp. (diam. $25 \mathrm{~cm}$, decay stage 3-4), in old-growth mesic heath forest, 11 Sep 2008, leg. PH 1781 (OULU), det. HK.

New to Middle Boreal, Northern Carelia - Kainuu (3b). This is the fourth record in Finland. Previous records are from Rovaniemi (3c, on Picea abies), Kuusamo (4a), Savukoski (4c, on P. abies) (Kotiranta et al. 2009). 


\section{Sidera vulgaris (Fr.) Miettinen}

Specimens examined: Regio aboënsis, Kemiönsaari, Örö, Storviken, UCS 6642:3238, on piece of conifer trunk (diam. $6 \mathrm{~cm}$, decay stage 4), in mixed mesic heath forest, 3 Oct 2015, leg. PK 9210 (TUR), det. JP; Nylandia, Helsinki, Hallainvuori, UCS 6681040:3391755, on fallen trunk of Pinus sylvestris (diam. $10 \mathrm{~cm}$, decay stage 3), in spruce-dominated mesic heath forest with quite high amount of dead wood, 1 Oct 2014, leg. KS T/011014 (H), det. V. Spirin; Helsinki, Haltiala SE-part, UCS 6685001:3385806, on fallen trunk of Picea abies (diam. $28 \mathrm{~cm}$ ), in old-growth mixed herb-rich forest with high amount of dead wood, 29 Oct 2014, leg. B. Weijola BW7/291014 (H), det. V. Spirin. These two latter records were earlier identified as Sidera "mitis".

There are five previous findigs from Finland: Nauvo (1b), Kirkkonummi (1b), Helsinki (Kivikko 1b, Haltiala 2a), and Sipoo (2a). (Vauras 2000, Kunttu et al. 2014, Savola 2015, Savola \& Kolehmainen 2015). Basidiomata grew on Picea abies, Pinus sylvestris, and Populus tremula. Not Evaluated.

Sistotrema autumnale Ryvarden \& H. Solheim

Specimen examined: Ostrobottnia kajanensis, Kajaani, Lahnasjärvi, Karsikkovaara, UCS 7102152:3539973, on fallen branch of Betula sp. (diam. $5 \mathrm{~cm}$, decay stage 2-3), in mesic herb-rich forest with a high degree of hemeroby, 28 Sep 2008, leg. PH 1800 (OULU), det. MK, conf. HK.

New to Middle Boreal, Northern Carelia - Kainuu (3b). This is the second record in Finland. The first record is from Padasjoki (2a), on Betula sp. (Kotiranta et al. 2009).

\section{Sistotrema dennisii Malencon}

Specimens examined: Regio aboënsis, Kemiönsaari, Örö, west shore, UCS 6642:3237, on conifer drift wood $\log$ (diam. $20 \mathrm{~cm}$, decay stage 2, length $3.1 \mathrm{~m})$, on sand beach, 2 Oct 2015, leg. PK 8935 (TUR), det. MK; Kemiönsaari, Örö, west shore, UCS 6641:3237, on conifer drift wood $\log$ (diam. 24, decay stage 2, length $5.3 \mathrm{~m}$ ), on sand beach, 3 Oct 2015, leg. PK 8957 (TUR), det. MK.

New to Hemiboreal, Oak zone (1b). This is the ninth record in Finland. Previous records derive throughout Finland (Kotiranta et al. 2009, Kunttu et al. 2013, 2014). Most Finnish records are from Pinus sylvestris, but also from Alnus incana and Picea abies. Data Deficient.

\section{Sistotrema resinicystidium Hallenb.}

Specimen examined: Ostrobottnia ultima, Rovaniemi, Pisavaara Strict Nature Reserve, UCS 7359396:3416486, on piece of fallen Populus tremula (diam. $30 \mathrm{~cm}$, decay stage 3), in old-growth mesic heath forest, 17 Sep 2015, leg. MK 74/15 \& PH (OULU), det. MK.

New to Middle Boreal, Southwestern Lapland (3c).

\section{Spiculogloea occulta P. Roberts}

Specimens examined: Ostrobottnia kajanensis, Kajaani, Suvantola, Ensilä UCS 7126564:3534602, on dead branch of Sambucus racemosa/Lyomyces sambuci, (diam. $3 \mathrm{~cm}$, decay stage 3) and on fallen trunk of Sambucus racemosa/Lyomyces sambuci (diam. 1,5 cm, decay stage 4), in herb-rich deciduous forest beside a brook, 8 Sep 2008, PH 1751, 1753 (OULU), det. HK \& MK.

New to Middle Boreal, Northern Carelia - Kainuu (3b).

Steccherinum collabens (Fr.) Vesterh.

Specimens examined: Ostrobottnia kajanensis, Vuolijoki, Patjamäki, UCS 7115712:3490916, on fallen trunk of Picea abies (diam. $14 \mathrm{~cm}$, decay stage 4), in old-growth forest, 7 Nov 2004, PH 1387 (OULU), det. MK.

New to Middle Boreal, Ostrobothnia (3a). Near Threatened. 
Steccherinum tenuispinum Spirin, Zmitr. \& Malysheva

Specimen examined: Nyland, Helsinki, Vuosaari, artificial hill N, UCS 6681341:3398101, on stump of Picea abies (diam. $40 \mathrm{~cm}$, decay stage 3, on same stump also Fomitopsis pinicola and Antrodiella citrinella), in old-growth forest with plenty of woody debris, 14 Oct 2014, leg. KS H/141014 (H), det. V. Spirin.

This is the sixth locality in Finland. Previous records are from Helsinki (Patola, Santahamina, Meri-Rastila, Vartiosaari; 1b) and Lammi (2a) (Miettinen 2012, Miettinen et al. 2012, Savola 2012, 2015), altogether on 11 trunks of mainly Picea abies or Alnus incana.

\section{Stypella vermiformis (Berk. \& Broome) D.A. Reid}

Specimens examined: Regio aboënsis, Kemiönsaari, Dragsfjärd, Gärskär, UCS 6645:3222, on four drift wood $\log$ (diam. $15 \mathrm{~cm}$, decay stage 4; diam. $15 \mathrm{~cm}$, decay stage 4; diam. $17 \mathrm{~cm}$, decay stage 3; diam. $11 \mathrm{~cm}$, decay stage 1), in sea shore meadow, 4 Sep 2015, leg. PK 8717a (OULU), 8720, 8722a, 8724b (TUR), det. MK; Kemiönsaari, Örö, west shore, UCS 6641:3237, on drift wood log of Picea abies (diam. 36 cm, decay stage 2), on sand beach, 4 Oct 2015, leg. PK $9126 \mathrm{~b}$ (TUR), det. MK.

New to Hemiboreal, Oak zone (1b). This is the fourth record in Finland. Previous records are from Lieksa (3b, two sites) and Inari (4c): species grew on Pinus sylvestris in all cases (Kotiranta et al. 2009).

Thanatephorus fusisporus coll. (J. Schröt.) Hauerslev \& P. Roberts

Specimen examined: Ostrobottnia kajanensis, Suomussalmi, Hossa, YKJ 71630:36237, fallen trunk of Populus tremula (diam. 13 cm, decay stage 4), in old-growth forest, 17 Oct 2015, PH 2948 (OULU, H.K.), det. HK.

New to Middle boreal, Northern Carelia - Kainuu (3b).

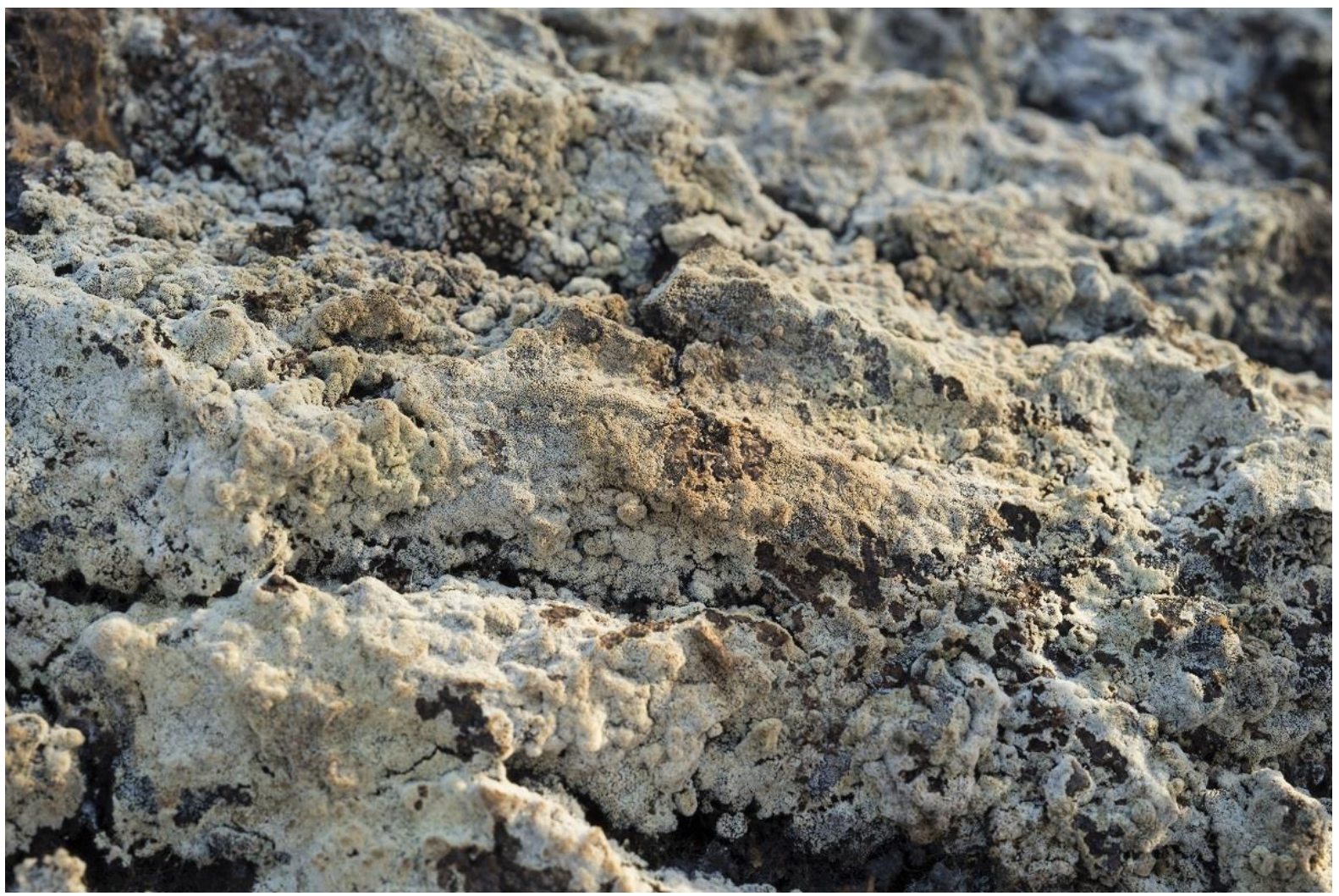

Fig. 7 - Thanatephorus fusisporus coll. on fallen trunk of Populus tremula in Suomussalmi (PH 2948). Photo: Pekka Helo. 
Thelephora caryophyllea Schaeff. : Fr.

Specimen examined: Ostrobottnia ultima, Keminmaa, church village, church, UCS 73043:33879, on ground in seminatural dry grassland, 30 Aug 2010, leg. \& det. E. Ohenoja (OULU), conf. MK.

New to Middle Boreal, Southwestern Lapland (3c).

Tomentella fibrosa (Berk. \& M.A. Curtis) Kõljalg

Specimen examined: Alandia, Lemland, Vesterbergen Nature Reserve, UCS 6679699:3108363, on fallen trunk of Picea abies (diam. $10 \mathrm{~cm}$, decay stage 3), in herb-rich heath forest, 23 Sep 2015, leg. JP 2990 (H, J.P.).

New to Hemiboreal, Åland (1a).

Tomentella radiosa (P. Karst.) Rick

Specimen examined: Ostrobottnia ultima, Tervola, Pisavaara Strict Nature Reserve, Vähäloma, UCS 7353457:3412994, on fallen trunk of Pinus sylvestris (diam. $30 \mathrm{~cm}$, decay stage 1), in old-growth xeric heath forest, 4 Sep 2015, leg. MK 19/15 (OULU).

New to Middle Boreal, Southwestern Lapland (3c).

\section{Tomentella subclavigera Litsch.}

Specimen examined: Ostrobottnia ultima, Rovaniemi, Pisavaara Strict Nature Reserve, UCS 7359391:3416521, on fallen trunk of Picea abies (diam. $30 \mathrm{~cm}$, decay stage 3), in old-growth mesic heath forest, 17 Sep 2015, MK 73/15 \& PH (OULU), det. MK.

New to Middle Boreal, Southwestern Lapland (3c). This is the sixth record in Finland. Previous records are from Helsinki (1a, three sites), Tammela (2a), and Savonranta (2b). Host trees were Salix sp., Alnus sp. and Populus tremula (Kotiranta et al. 2009).

Tomentellopsis submollis (Svrček) Hjortstam

Specimen examined: Ostrobottnia ouluensis, Oulu, Sanginjoki, Isokangas, UCS 7212890:3447087, on fallen trunk of Pinus sylvestris (diam.16 cm, decay stage 2), in xeric heath forest, 18 Oct 2015, MK 107/15 (OULU).

New to Middle Boreal, Ostrobothnia (3a).

Trechispora cohaerens (Schw.) Jülich \& Stalpers

Specimen examined: Ostrobottnia ultima, Tervola, Pisavaara Strict Nature Reserve, Pihlajakurunlampi, UCS 7353916:3412122, on fallen trunk of Betula sp. (diam. $23 \mathrm{~cm}$, decay stage 4), in old-growth mesic heath forest, 4 Sep 2015, leg. MK 21/15 (OULU).

New to Middle Boreal, Southwestern Lapland (3c).

\section{Trechispora elongata Miettinen \& K. H. Larsson}

Specimen examined: Regio aboënsis, Kemiönsaari, Örö, west shore, UCS 6642:3237, on drfit wood $\log$ of Pinus sylvestris, (diam. $21 \mathrm{~cm}$, decay stage 4, length $3.8 \mathrm{~m}$ ), on sand beach, 1 Oct 2015, leg. PK 8737 (TUR), det. MK.

New to Hemiboreal, Oak zone (1b). This is the second Finnish record. The first record is from Jyväskylä (2b), on Populus tremula (Miettinen \& Larsson 2006).

Trechispora laevis K.H. Larss.

Specimen examined: Regio aboënsis, Kemiönsaari, Dragsfjärd, Örö, west shore, UCS 6642:3237, on unidentified drift wood $\log$ (diam. $14 \mathrm{~cm}$, decay stage 4 , length $4 \mathrm{~m}$ ), on sand beach, 2 Oct 2015, leg. PK 8913 (TUR), det. MK.

This is the tenth record in Finland. Previous records derive throughout Finland (Kotiranta et al. 2009, Kunttu et al. 2009, 2012, 2014, 2015). 
Trechispora microspora (P. Karst.) Liberta

Specimen examined: Lapponia inarensis, Inari, Vätsäri Wilderness Area, Auttojänkä, UCS 7660636:3552153, on broken fallen trunk of Betula sp. (diam. 22, decay stage 4), 13 Aug 2009, leg. PK 4914 (TUR), det. JP.

New to Northern Boreal, Forest Lapland (4c).

Trechispora silvae-ryae (J. Erikss. \& Ryvarden) K.H. Larss.

Specimen examined: Kemiönsaari, Dragsfjärd, Örö, west shore, UCS 6641:3237, on drfit wood log of Picea abies (diam. $23 \mathrm{~cm}$, decay stage 2, length $3.1 \mathrm{~m}$ ), on shore meadow, 4 Oct 2015, leg. PK 9104 (TUR), det. MK, conf. HK.

This is the second record from Finland. The first record is from Helsinki (1b) on Alnus incana, but this site is destroyed (Kotiranta \& Saarenoksa 2000). Data Deficient.

Trechispora stevensonii (Berk. \& Broome) K.H. Larss.

Specimens examined: Ostrobottnia kajanensis, Puolanka, Paljakka, Mustakumpu, Röllilampi, UCS 7172609:3550619, on cut log of Picea abies? (diam. $4.5 \mathrm{~cm}$ decay stage 5), in old-growth mesic heath forest, 30 Sep 2011, PH 2053 (OULU), det. MK; Kajaani, Suvantola, UCS 7126987:3533930, on rootplate of deciduous tree (diam. $30 \mathrm{~cm}$, decay stage 4), in mesic herb-rich forest with a high degree of hemeroby, 2 Nov 2011, PH 2200, (OULU), det. MK.

New Middle boreal, Northern Carelia - Kainuu (3b).

Tremella polyporina D.A. Reid

Specimen examined: Ostrobottnia kajanensis, Hyrynsalmi, Kytömäki, Iso Tuomivaara, UCS7188796:3558948, in basidiome of Postia sp. which grew on root of Picea abies (diam. $20 \mathrm{~cm}$, decay stage 2), in mesic heath forest, 3 Sep 2012, leg. PH 2309 (OULU), det. MK.

New Middle boreal, Northern Carelia - Kainuu (3b). This is the fifth record in Finland. Previous records are from Helsinki (1b), Lammi (2a), Tampere (2a) and Inari (4c). In all cases $T$. polyporina grew inside of polypore's basidiome (Kotiranta et al. 2009).

Tubulicrinis hirtellus (Bourdot \& Galzin) J. Erikss.

Specimen examined: Ostrobottnia ultima, Tervola, Pisavaara Strict Nature Reserve, UCS 7353715:3412598, on fallen trunk of Pinus sylvestris (diam. $10 \mathrm{~cm}$, decay stage 2), in rocky and barren heath forest, 4 Sep 2015, leg. MK 20/15 (OULU).

New to Middle Boreal, Southwestern Lapland (3c). This is the third record in Finland. Previous record are from Lammi (2a) and Kuhmo (3b), on Pinus sylvestris (Kotiranta et al. 2009).

Tulasnella albida Bourdot \& Galzin

Specimen examined: Ostrobottnia ouluensis, Oulu, Sanginjoki, Isokangas, UCS 7212040:3447262, fallen trunk of Betula sp. (diam. $3.5 \mathrm{~cm}$, decay stage 1), in mesic heath forest, 18 Oct 2015, MK 111/15 (OULU), conf. HK.

New to Middle Boreal, Ostrobothnia (3a). This is the seventh record in Finland. Previous records are from Sipoo (2a, on Abies sibirica cult.), Padasjoki (2a, on Betula sp.), Pieksänmaa (2b, on Populus tremula), and Utsjoki (4d, on Salix sp.) (Kotiranta et al. 2009, Kotiranta \& Shiryaev 2013). Additionally, there are records from two sites in Central Finland (Juutilainen et al. 2014).

Tulasnella subglobispora Hjortstam

Specimens examined: Ostrobottnia ouluensis, Muhos, Hyrkäs, UCS 7190506:3458245, on fallen trunk of Populus tremula (diam. $7 \mathrm{~cm}$ ), in mesic herb-rich forest, 25 Sep 2000, M. Ohenoja 3 (OULU), det. MK, conf. HK; Ostrobottnia ultima, Rovaniemi, Pisavaara Strict Nature Reserve, Teeripäri, UCS 7358602:3414704, on stump of conifer (diam. $35 \mathrm{~cm}$, decay stage 1), in old-growth mesic heath forest, 3 Oct 2015, leg. MK 90/15 (OULU).

New to Middle Boreal, Ostrobothnia (3a) and Middle Boreal, Southwestern Lapland (3c). 
Typhula erythropus (Pers.:Fr.) Fr.

Specimen examined: Ostrobottnia kajanensis, Kajaani, Lehtovaara, UCS 71090:35334, in eutrophic mixed forest with Picea abies, Sorbus aucuparia, Betula spp. and Alnus incana, 14 Sep 2015, leg. TH 150022 (OULU).

New to Middle boreal, Northern Carelia - Kainuu (3b).

Typhula uncialis (Grev.:Fr.) Berthier

Specimen examined: Ostrobottnia kajanensis, Sotkamo, Rikkola, Känttäjä, UCS 71063:35474, on thicket of Rubus idaeus in field dominated by Epilobium angustifolium, 21 Sep 2015, leg. TH 150023 (OULU).

New to Middle boreal, Northern Carelia - Kainuu (3b).

Tyromyces fumidiceps G.F. Atk.

Specimen examined: Ostrobottnia ouluensis, Oulu, Vihriäsaari, UCS 7213757:3425986, on drift wood? $\log$ (diam. $30 \mathrm{~cm}$, decay stage 4), in seasonally wet birch-willow thicket, 2 Sep 2011, MK 22/11 (OULU), det. V. Spirin \& T. Niemelä. This specimen was identified earlier as Tyromyces canadensis Overh. ex J. Lowe and published by Kunttu et al. (2012).

New to Middle Boreal, Ostrobothnia (3a). This is the fourth record in Finland. Previous records are from two sites in Kirkkonummi (1b) and Tammisaari (1b). Basidiomata grew on Alnus glutinosa and unidentified deciduous tree (Renvall \& Kaaro 1998, Kotiranta et al. 2009). Endangered.

Vuilleminia comedens (Nees : Fr.) Maire

Specimen examined: Ostrobottnia kajanensis, Kajaani, Suvantola, UCS 7127025:3533842, on fallen trunk of Alnus incana (diam. $3.5 \mathrm{~cm}$, decay stage 3), in herb-rich forest with a high degree of hemeroby, 4 Nov 2011, PH 2213 (OULU), det. MK

New Middle boreal, Northern Carelia - Kainuu (3b).

Xylodon nespori (Bres.) Hjortstam \& Ryvarden

Specimens examined: Ostrobottnia kajanensis, Sotkamo, Parkua, Känttäjä, UCS 7106333:3547370, on coniferous timber plank board, (decay stage 2), in yard, 17 Sep 2009, PH 1868 (OULU), det. MK; and in same place, on timber plank board, 8 Nov 2011, PH 2245 (OULU), det. MK.

This is the fifth record in Finland. Previous records are from Kemiönsaari (1b, on Quercus robur), Suomussalmi (3b, on Pinus sylvestris) and two localities in Kuusamo (4a, on P. sylvestris) (Kotiranta et al. 2009, Kunttu et al. 2015).

\section{Discussion}

\section{Species new to Finland}

Ceriporia bresadolae is globally a rare species found from France, Spain, the United States (Bourdot \& Galzin 1925, Gilbertson et al. 1974, Martinez-González \& Honrubia 1985), and Russia (H. Kotiranta, unpubl.). It is macroscopically and microscopically very similar to $C$. purpurea, but it grows on hard coniferous trees, often on Pinus, like Pinus halepensis and Pinus ponderosa (Gilbertson 1974, Martinez-González \& Honrubia 1985). All Finnish records are from Pinus sylvestris, two of them on timber plank board.

Clavaria tenuipes is presented here as new to Finland. Because of taxonomical uncertainty, it was not mentioned in the checklist of aphyllophoroid fungi of Finland (Kotiranta et al 2009) even there is one published record from Kuusamo (4a) in 1978, and specimen is deposited in herbarium of Trondheim, Norway (Sievertsen et al. 1994). Records presented are mostly confirmed by Ivona Kautmanová from Bratislava. Here $C$. tenuipes is considered as in Funga Nordica (Knudsen \& Vesterholt 2008): pale greyish buff chraceous basidiomata with ellipsoid spores and basidia with loop-like clamps. Distribution area is large covering the Middle and Northern parts of Europe and 
Russia, Sverdlovsk Region in Ural Mountains (Shiryaev et al. 2010). For example, there are many collections from Czech Republic and Slovenia (I. Kautmanová, unpubl.). Clavaria tenuipes is probably not an uncommon species in Finland, but further studies are needed.

Renatobasidium notabile is an extremely rare species in the world and before this Finnish record, it was reported only from Denmark where it grew probably on Alnus (Hauerslev 1993). We found no more information about ecology or distribution of this species. Finnish growing site is situated in the south coast and the substratum was Juniperus communis.

\section{Species with only a few previous records from Finland}

Antrodia cretacea is known from three localities in Finland, and globally species occurs in temperate and boreal zones of Europe, East Asia, and North America. In Europe its distribution is more southern than that of A. crassa, and it preferably grows on lying trunks or stumps of Picea spp. (Spirin et al. 2015). Antrodia leucaena is found four times from Southern Finland, and globally there are findings from Russia and China, substrata were mainly Populus spp. (Spirin et al. 2013, Dai \& Niemelä 2002).

All three Finnish records of Ceriporia aurantiocarnescens are from Helsinki (1b), on deciduous trees. In Europe, the species is found from France and Spain, and substrata were deciduous trees, such as Acer, Fagus and Populus (Ryvarden \& Melo 2014).

During summer and autumn 2015 seven new records of Clavaria flavipes were made from Middle and Northern boreal zones. Previous this species was known from two sites from Southern Finland. A good year for clavarioid fungi and active field work proved $C$. flavipes to be common and wide spread in Finland. Furthermore, in addition to these records there are some unpublished record from Southern Finland (S. Jakobsson, pers. comm.).

Both records of Crustomyces subabruptus are from Finnish SW-archipelago. In Europe, it occurs through the continent, and seems to be common in Central Europe. The species occurs in dry and warm habitats, both on coniferous and deciduous wood, like Pinus sylvestris, Populus tremula, Fagus sylvatica and Carpinus spp. (Eriksson \& Ryvarden 1975, Bernicchia \& Gorjón 2010).

Phanerochaete livescens is a widespread species in temperate Eurasia (Volobuev et al. 2015), but there are only three records from Finland: Helsinki (1b), Tammela (2a) and Ilomantsi (2b), and Pinus sylvestris was the host tree in all records. The first Finnish record dates back until 1894 (Karsten 1895). The species was forgotten for a long time and included in P. sordida (P. Karst.) J. Erikss. \& Ryvarden (Volobuev et al. 2015). Re-identification of Finnish herbarium material in genus Phanerochaete might reveal new records.

Sistotrema autumnale is now found from Padasjoki (2a) and Kajaani (3b) in Finland. In both cases the substratum was Betula sp. According to Bernicchia \& Gorjón (2010) there are records from France, Germany, Sweden, Norway and Czech Republic. S. autumnale is collected from decayed deciduous trees: Alnus, Betula, Salix, and Sorbus (Eriksson \& Ryvarden 1975).

Trechispora elongata is now found from Kemiönsaari (1b) and Jyväskylä (2b) in Finland. Miettinen \& Larsson (2006) reported six records from the Nordic countries and one from Poland. All specimens grew on strongly decomposed wood, and six specimens grew on Picea abies or Pinus sylvestris, one on Populus tremula. Habitats varied in their quality, but mostly they seem to be managed coniferous forests. Although inconspicuous and thus easily overlooked, the species seems to be rather rare (Miettinen \& Larsson 2006).

Both Finnish records of Trechispora silvae-ryae derive from south coast, Kemiönsaari (1b) and Helsinki (1b). It is defined as a rare species, but distribution in Europe covers also United Kingdom, Spain, Italy, Denmark, Sweden, Czech Republic, France, and Germany. Additionally it is found from nemoral areas in Canada and Himalaya (Larsson 1992, Bernicchia \& Gorjón 2010). T. silvae-ryae grows on Populus, Quercus ilex, other deciduous wood, litter in pine forest, and Pinus pinea (Bernicchia \& Gorjón 2010). According to Larsson (1992) it grows on a variety of well decayed woody substrata, but also on herbaceous material like Carex pendula and on dead basidiomata of polypores. 
The records of Tubulicrinis hirtellus are scattered through Finland: Lammi (2a), Kuhmo (3b) and Tervola (3c), in all cases the substratum was Pinus sylvestris. It is considered as a rare species and in addition to these Finnish records, records in Europe are made from Sweden, Germany, Italy, Norway, Spain, and Austria from stumps of Picea abies and Larix decidua and on lying trunk of Abies alba (Bernicchia \& Gorjón 2010).

\section{Notes on substrata}

According to Kotiranta et al. (2009) Phlebiella christiansenii grows mainly on deciduous wood in Finland, but now it was found from Picea abies.

The substrata of Steccherinum tenuispinum (8 units) found in Helsinki, were strongly decayed by Fomitopsis pinicola and four of them was accompanied with Antrodiella citrinella (Savola 2012, 2015). This interaction was mentioned also by Spirin et al. (2007).

Oligoporus romellii is not so rare in Finland as these published records may show. It is relatively recently described species (Pieri \& Rivoire 2006) and earlier it was not separated from Oligoporus sericeomollis. It may have southern distribution in Finland and it grows mainly on conifers (K. Savola, unpubl.), in contrast to Niemelä (2012). Re-identification of herbarium material of $O$. sericeomollis would probably reveal much more records of $O$. romellii.

According to our records, substrata of Antrodia cretacea and Antrodia piceata were strongly decayed by Fomitopsis pinicola. This interaction was mentioned in Kotiranta \& Niemelä (1996) in case of Antrodia sitchensis, i.e. now known as A. piceata. These kinds of successor and their predecessor relations with $F$. pinicola was recognized for example with Antrodiella citrinella and Pycnoporellus fulgens (Niemelä et al. 1995). Similar interactions with Pycnoporellus alboluteus and Steccherinum tenuispinum are maybe not so strict but exist anyway very often (see, Niemelä 2005). Most records of A. piceata are from old-growth forests, where it inhabits large lying trunks of conifer trees, mostly Picea spp. (Spirin et al. 2015).

Several records in current paper derived from kelo trees. In general, kelo trees are known to be crucial substrata for some rare and threatened wood-inhabiting fungi. Kelo trees are special substrata for fungi due their long formation history, age, surface and chemical compounds (Niemelä et al. 2002, Venugopal et al. 2016).

\section{Notes on geographical extensions}

Most of the new records are from regions where they were expected, relatively close to their formerly known area of occupancy. However, the records of some species are far from their earlier finds or at least in a surprising direction. One very obvious explanation of the "expansion" of southern species to the north is the increasing collecting activity in Northern and Eastern Finland.

Byssocorticium pulchrum was found now from Rovaniemi (3c) but previous records are from Southern Finland. The new record of Clavaria amoenoides is the northernmost in Finland (Kotiranta et al. 2009). Cystostereum murraii was found now from Åland (1b), but its main occurrence area is currently in Northern Finland, and it occurs only sporadically between these areas. Luellia recondita was now found from Rovaniemi (3c) in Northern Finland, but all previous records are from Finnish south coast. The distance to the nearest previous record was over $600 \mathrm{~km}$.

So far, Ramariopsis kunzei was found only from Southern Finland and new the record from Rovaniemi is $600 \mathrm{~km}$ north of the old ones. Spiculogloea occulta was found this time from Kajaani (3b), and the previous records are from Southern Finland (1b and 2a) (Kotiranta et al. 2009). The new record of Stypella vermiformis from Kemiönsaari (1b) is $550 \mathrm{~km} \mathrm{SW}$ from the nearest previous known occurrence in Lieksa (3b). Tomentella subclavigera was found from Rovaniemi (3c), but all previous records are from Southern and Eastern Finland about $500 \mathrm{~km}$ from the new one. Tyromyces fumidiceps was found from Oulu (northern part of 3a) and this is over $500 \mathrm{~km}$ north from previous records from Finnish south coast. However, all these records are from coast area of the Baltic Sea.

There are now only six records of Sidera vulgaris in Finland, all from southern coast, but in Estonia it is a fairly common species, at least on island of Hiiumaa with wide range of forest 
habitats, both on conifers and deciduous trees (J. Pennanen, unpublished). It is possible that in the future it will become more common in Finland as well and maybe the global warming of the climate also affects this phenomenon. According to Finnish collections of Resinicium pinicola, it seems to be very rare and it maybe has a maritime distribution.

\section{Little collected species, but probably not so rare as thought}

Our results include some species which are little collected and therefore considered as rare. These species are little collected due to their tiny or difficult-to-detect basidiomata, or are challenging to identify. Colacogloea peniophorae is an example and is apparently much more common than the hitherto records show. It is often overlooked when quickly identifying the host species. The most important host is very common Peniophorella praetermissa. The specimens of Luellia recondita are often found accidentially when the purpose was to collect a specimen of some other species. Basidiomata are small and colour does not stand out on substrata. According to our experience Sistotrema dennisii grows often on small pieces of dead wood and this kind of substratum is often overlooked in general species inventories. It is maybe not so rare, at least in Finland, as stated (Kotiranta et al. 2009, Ryvarden \& Melo 2014). Spiculogloea occulta and Tremella polyporina are mycoparasites with invisible basidiomata and therefore collected only accidentally.

\section{Notes on clavarioid species}

The year 2015 was exceptionally good for fungi of cultural habitats in northern parts of Finland. Plenty of waxcaps, clavarioid and geoglossoid fungal records were made from dry grasslands, grazing grounds, roadsides, lawns and cemeteries. Rainy summer 2015 was one reason for this phenomenon. Clavaria amoenoides, C. flavipes, $C$. greletii and C. rosea, which have earlier been concidered as uncommon or rare, are shown to be rather common and to have a wide distribution area in Finland. Clavaria greletii is also difficult to notice because of small size and dark grey colour. Moreover, it produces basidiomata only in appropriate circumstances.

Clavarioid taxa have been often overlooked in northern parts of Finland. Reasons for that are the lack mycologists working in the north and the very unpredictable occurence of clavarioid fungi. There are also many taxonomical problems with clavarioid and ramarioid fungi. Some of the specimens collected during summer 2015 are still without valid names. Problems concern mostly species groups Clavaria incarnata coll., Ramariopsis subtilis coll. and recently described Clavaria flavostellifera (Olariaga et al. 2015), which are not presented here.

\section{Concluding remarks}

The distribution of aphyllophoroid fungi is still inadequately known in many regions in Finland, especially in northern and eastern parts, and in southwestern archipelago, as shown in this article. However, new noteworthly records are reported frequently and therefore knowledge accumulates all the time (e.g. Savola \& Kolehmainen 2015, Juutilainen 2016, Kunttu et al. 2016). It is obvious that dozens of species can still be found in these areas, so that the ranges of the species could be less fragmented (on a large scale) than it is in the distribution maps (Kotiranta et al. 2009). Most likely there are numerous sites where many of these little collected species occur between the scattered current records. To establish which species are truly rare, more effort should be put into field surveys. Of course a certain portion of aphyllophoroid fungi are truly rare, for example because of their specialisation in a certain habitat or substratum.

\section{Acknowledgements}

We are grateful to many persons who have helped us. Pekka Helo kindly provided us a photograph of Thanatephorus fusisporus coll. and made several collections. Tuomas Kallio collected the specimen of Ceriporia bresadolae. Sanna-Mari Kunttu drew the map and assisted in the field work. Additionally, the following persons made collections or identified specimens: Slavomir Adamčík, Pekka Halonen, Emma Heinänen, Stefan Jakobsson, Ivona Kautmanova, Panu 
Keihäs, Juha Kinnunen, Miia Kokkonen, Heino Kulju, Otto Miettinen, Tuomo Niemelä, Esteri Ohenoja, Martti Ohenoja, Anna-Liisa Ruotsalainen, Viacheslav Spirin, Tiiu Tenno, Maarit Tyyskä, Jarmo Uimonen, and Birthe Weijola.

\section{References}

Anonymous 2014 - Eliömaakunnat [Biogeographical provinces of Finland]. Finnish Museum of Natural History, http://www.luomus.fi/fi/eliomaakunnat. (Accessed 23 Feb 2016)

Bernicchia A, Gorjón SP. 2010 - Corticiaceae s. 1. Fungi Europaei. Vol. 12, Edizioni Candusso, Alassio, 1008 pp.

Boddy L, Frankland JC, van West P. 2008 - Ecology of saprotrophic basidiomycetes. London, Elsevier Academic Press, 386 pp.

Bourdot H, Galzin A. 1925. Hyménomycètes de France XI. Porés. - Bulletin de la Société Mycologique de France. 41, 98-255.

Dai YC, Niemelä T 2002. - Changbai wood-rotting fungi 13. Antrodia sensu lato. Annales Botanici Fennici 39, 257-265.

Dudka IO, Romanenko KO. 2006 - Co-existence and interaction between myxomycetes and other organisms in shared niches. Acta Mycologica 41, 99-112.

Eriksson J, Ryvarden L. 1975 - The Corticiaceae of North Europe 3: Coronicium - Hyphoderma. Fungiflora, Oslo, pp. 288-546.

Gilbertson RL. 1974. - Fungi that decay ponderosa pine. University of Arizona Press, 197 pp.

Gilbertson RL, Martin KJ, Lindsey JP. 1974. - Annotated check list and host index for Arizona wood-rotting fungi. Bulletin (University of Arizona. Agricultural Experiment Station) 209, $1-48$.

Hauerslev K. 1993 - New tremellaceous fungi from Denmark. Mycotaxon 49, 217-233.

Heikinheimo O, Raatikainen M. 1981 - Grid references and names of localities in the recording of biological finds in Finland. Notulae Entomologicae 61, 133-154. (In Finnish with English summary)

Hibbett DS, Bauer R, Binder M, Giachini AJ, Hosaka K, Justo A, Larsson E, Larsson KH, Lawrey JD, Miettinen O, Nagy LG, Nilsson RH, Thorn RG. 2014 - 14 Agaricomycetes. In: Systematics and Evolution, 2nd Edition. Springer-Verlag, Berlin Heidelberg, pp. 373-429.

Hjortstam K, Ryvarden L. 2009 - A checklist of names in Hyphodontia sensu stricto - sensu lato and Schizopora with new combinations in Lagarobasidium, Lyomyces, Kneiffiella, Schizopora and Xylodon. Synopsis Fungorum 26, 33-55.

Jonsell M, Nordlander G, Ehnström B. 2001 - Substrate preferences of insects breeding in wood decaying fungi. Ecological Bulletins 49, 173-194.

Juutilainen K. 2016 - Ecology, environmental requirements and conservation of corticioid fungi occupying small diameter dead wood. Jyväskylä Studies in Biological and Environmental Science 313, 1-55.

Juutilainen K, Mönkkönen M, Kotiranta H, Halme P. 2014 - The effects of forest management on wood-inhabiting fungi occupying dead wood of different diameter fractions. Forest Ecology and Management 313, 283-291. DOI: 10.1016/j.foreco.2013.11.019

Karsten PA. 1895 - Fragmenta mycologica. XLIII. Hedwigia 34, 7-9.

Kekki T. 2015 - Ruusunuijakas Suomessa. Sienilehti 67(3), 73-76. (In Finnish)

Knudsen H, Vesterholt J. (Eds.) 2008 - Funga Nordica. Agaricoid, boletoid and cyphelloid genera. Nordsvamp, Copenhagen, 965 pp.

Kotiranta H, Junninen K, Saarenoksa R, Kinnunen J, Kytövuori I. 2010 - Aphyllophorales \& Heterobasidiomycetes. - In: Rassi P, Hyvärinen E, Juslén A, Mannerkoski I. (Eds.), The 2010 Red List of Finnish Species. Ministry of the Environment \& Finnish Environment Institute, Helsinki, pp. 249-263.

Kotiranta H, Larsson KH. 2013 - Sistotrema luteoviride sp. nov. (Cantharellales, Basidiomycota) from Finland. Acta Mycologica 48, 219-222. DOI: 10.5586/am.2013.023. 
Kotiranta H., Niemelä T. 1996 - Threatened polypores in Finland. 2nd revised edition. Finnish Environment Institute \& Edita, Helsinki, 184 pp. (In Finnish with English summary)

Kotiranta H, Saarenoksa R. 2000 - Corticioid fungi (Aphyllophorales, Basidiomycetes) in Finland. Acta Botanica Fennica 168, 1-55.

Kotiranta H, Saarenoksa R, Kytövuori I. 2009 - Aphyllophoroid fungi of Finland. A check-list with ecology, distribution, and threat categories. Norrlinia 19, 1-223.

Kotiranta H, Shiryaev A. 2013 - Notes on aphyllophoroid fungi (Basidiomycota) in Kevo, collected in 2009. Kevo Notes 14, 1-22.

Kunttu P, Kosonen T, Kulju M, Kotiranta H. 2009 - Phlebia cremeoalutacea new to Finland and new records of rare corticioid fungi (Basidiomycota). Karstenia 49, 69-71.

Kunttu P, Kulju M, Kotiranta H. 2010 - Rare corticioid fungi in Finland - records of new and little collected species (Basidiomycota). Karstenia 50, 35-44.

Kunttu P, Kulju M, Kotiranta H. 2012 - New national and regional biological records for Finland 2. Contributions to the Finnish aphyllophoroid funga (Basidiomycota). Memoranda Societas Fauna Flora Fennica, 88, 61-66.

Kunttu P, Kulju M, Kotiranta H. 2015. - Contributions to the Finnish aphyllophoroid funga (Basidiomycota): new and rare species. Czech Mycology 67, 137-156.

Kunttu P, Kulju M, Kotiranta H. 2016 - Athelia singularis and Leptosporomyces mundus (Basidiomycota) new to Finland. Mycobiota 6, 29-37. DOI:10.12664/mycobiota.2016.06.03

Kunttu P, Kulju M, Pennanen J, Kotiranta H, Halme P. 2011 - Additions to the Finnish aphyllophoroid fungi. Folia Cryptogamica Estonica 48, 25-30.

Kunttu P, Pennanen J, Helo T, Kulju M, Söderholm U. 2013 - New national and regional biological records for Finland 4. Additions to the knowledge of Finnish aphyllophoroid funga (Basidiomycota). Memoranda Societas Fauna Flora Fennica 89, 119-124.

Kunttu P, Pennanen J, Kulju M, Kekki T, Suominen M. 2014 - Noteworthy records of aphyllophoroid fungi in Finland (Basidiomycota). Acta Mycologica 49, 221-235. DOI: 10.5586/am.2014.017.

Larsson KH. 1992 - The genus Trechispora (Corticiaceae, Basidiomycetes). Ph.D. thesis, University of Göteborg, Göteborg.

Leikola M. 1969 - On the termination of diameter growth of Scots pine in old age in northernmost Finnish Lapland. Silva Fennica 3, 50-61. (In Finnish with English summary)

Martinez-González MC, Honrubia M. 1985 - Respiración endógena de Ceriporia bresadolae (Bdot. et Galz.) Bond. \& Sing. Aphyllophorales: Basidiomycotina. Anales de Biologia 5, 49-51.

Miettinen O. 2012 - The Corticiaceae survey of the forests in Veräjämäki, Patola and Tali 2011. Publications by City of Helsinki Environment Centre 6/2012, 24 pp. (In Finnish)

Miettinen O, Larsson E, Sjökvist E, Larsson KH. 2012 - Comprehensive taxon sampling reveals unaccounted diversity and morphological plasticity in a group of dimitic polypores (Basidiomycota, Polyporales). Cladistics 28, 251-270. DOI: 10.1111/j.10960031.2011.00380.x

Miettinen O, Larsson KH. 2006 - Trechispora elongata species nova from North Europe. Mycotaxon, 96, 193-198.

Miettinen O, Larsson KH. 2011 - Sidera, a new genus in Hymenochaetales with poroid and hydnoid species. Mycological Progress 10, 131-141. DOI: 10.1007/s11557-010-0682-5.

Niemelä T. 2005 - Polypores, lignicolous fungi. Norrlinia 13, 1-319. (In Finnish with English summary)

Niemelä T. 2012 - Guide to the polypores of Finland. Botanical Bulletins of the University of Helsinki 194, 1-118. (In Finnish, keys in English)

Niemelä T, Renvall P, Penttilä R. 1995 - Interactions of fungi at late stages of wood decomposition. Annales Botanici Fennici 32, 141-152.

Niemelä T, Wallenius T, Kotiranta H. 2002 - The kelo tree, a vanishing substrate of specified wood-inhabiting fungi. Polish Botanical Journal 47, 91-101 
Olariaga I, Salcedo I, Daniëls PP, Spooner B, Kautmanová I. 2015 - Taxonomy and phylogeny of yellow Clavaria species with clamped basidia - Clavaria flavostellifera sp. nov. and the typification of C. argillacea, C. flavipes and C. sphagnicola. Mycologia 107, 104-122. DOI: $10.3852 / 13-315$

Pieri M, Rivoire B. 2006 - A propos du complexe Postia sericeomollis. Bulletin Mensuel de la Société Linnéenne de Lyon. 75(3), 113-133.

Rassi P, Hyvärinen E, Juslén A, Mannerkoski I. (Eds.) 2010 - The 2010 Red List of Finnish Species. Ministry of the Environment \& Finnish Environment Institute, Helsinki, 685 pp.

Renvall P. 1995 - Community structure and dynamics of wood-rotting Basidiomycetes on decomposing conifer trunks in Northern Finland. Karstenia 35, 1-51.

Renvall P, Kaaro J. 1998 - Tyromyces fumidiceps - an addition to the polypore flora of North Europe. Folia Cryptogamica Estonica 33, 123-126.

Ryvarden L, Melo I. 2014 - Poroid Fungi of Europe. Synopsis Fungorum 31, 1-455.

Savola K. 2012 - The polypore survey of the forests in Helsinki 2011. Publications by City of Helsinki Environment Centre 5/2012, 45 pp. (In Finnish)

Savola K. 2015: The polypore survey of the forests in Helsinki - supplement 2014. Publications by City of Helsinki Environment Centre 1/2015, 61 pp. (In Finnish)

Savola K, Kolehmainen K. 2015 - Pääkaupunkiseudun Viherkehän suojelualueiden käävät. Metsähallituksen luonnonsuojelujulkaisuja A 220, 1-65. (In Finnish)

Shiryaev AG, Kotiranta H, Mukhin, VA, Stavishenko IV, Ushakova NV. 2010 - Aphyllophoroid fungi of Sverdlovsk region, Russia: Biodiversity, distribution, ecology and the IUCN threat categories. Russian Academy of Science, Ural Division, Inst. Plant Animal Ecology, Ekaterinburg, 303 pp.

Sievertsen S., Jordal J.B. \& Gaarder G. 1994 - Noen soppfunn I ugjødsla beite- og slåttemarker. Agarica 13(22), 1-38. (In Norwegian)

Spirin V, Miettinen O, Pennanen J, Kotiranta H, Niemelä T. 2013 - Antrodia hyalina, a new polypore from Russia, and A. leucaena, new to Europe. Mycological Progress 12, 53-61. DOI: $10.1007 / \mathrm{s} 11557-012-0815-0$.

Spirin V, Runnel K, Vlasák J, Miettinen O, Põldmaa K. 2015 - Species diversity in the Antrodia crassa group (Polyporales, Basidiomycota). Fungal Biology 119, 1291-1310. DOI: 10.1016/j.funbio.2015.09.008

Spirin V, Zmitrovich I, Malycheva V. 2007 - Steccherinum tenuispinum (Polyporales, Basidiomycota), a new species from Russia, and notes on three other species. Annales Botanici Fennici 44, 298-302.

Stokland J, Sippola AL. 2004 - Monitoring protocol for wood-inhabiting fungi in the Alberta Biodiversity Monitoring Program. Alberta Biodiversity Monitoring Program, Alberta, 58 pp.

Vauras J. 2000 - Macrofungi of the Southwestern Archipelago National Park. Metsähallituksen luonnonsuojelujulkaisuja A 112, 1-91. (In Finnish with English summary)

Venugopal P, Julkunen-Tiitto R, Junninen K, Kouki J. 2016 - Phenolic compounds in Scots pine heartwood: are kelo trees a unique woody substrate? Canadian Journal of Forest Research 46, 225-233. DOI: 10.1139/cjfr-2014-0498

Volobuev S, Okun M, Ordynets A, Spirin V. 2015 - The Phanerochaete sordida group (Polyporales, Basidiomycota) in temperate Eurasia, with a note on Phanerochaete pallida. Mycological Progress 14, 80. DOI: 10.1007/s11557-015-1097-0 